\title{
Identification and Analysis of the Historical Origins of Ergonomics by Referenced Publication Year's Spectroscopy
}

\author{
Rashid Heidarimoghadam ${ }^{1}$, Ali Akbar Khasseh ${ }^{2}\left({ }^{\infty}\right)$ Hossein Vakilimofrad $^{3(D)}$, Abbas Fattahi $^{4}$, \\ Mohammad Reza Amiri* ${ }^{* *}$
}

1. Professor, School of Health, Hamadan University of Medical Sciences, Hamadan, Iran

2. Associate Professor, Department of Information Science and Knowledge Studies, Payame Noor University, Tehran, Iran

3. Assistant Professor, Department of Medical Library and Information Sciences, Hamadan University of Medical

Sciences, Hamadan, Iran

4. Department of Medical Library and Information Sciences, Hamadan University of Medical Sciences, Hamadan, Iran

\begin{tabular}{|c|}
\hline Article Info \\
\hline $\begin{array}{l}\text { Received: 2021/04/07; } \\
\text { Accepted: 2021/05/22; } \\
\text { ePublished: 2021/09/21 }\end{array}$ \\
\hline do) $10.30699 /$ jergon.9.2.42 \\
\hline $\begin{array}{l}\text { Use your device to scan } \\
\text { and read the article online }\end{array}$ \\
\hline 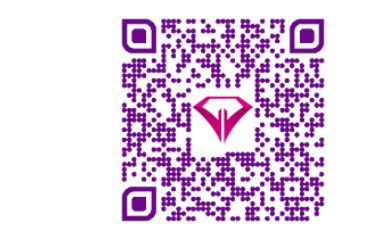 \\
\hline
\end{tabular}

Corresponding Author

Mohammad Reza Amiri

Assistant Professor,

Department of Medical

Library and Information

Sciences, Hamadan

University of Medical

Sciences, Hamadan, Iran

Email:

m.r.amirilib@gmail.com

\begin{abstract}
Background and Objectives: Every scientific activity needs to know the previous related scientific activities so that its various aspects can be better investigated in order to obtain a richer intellectual heritage. So this article aims to identify and analyze the historical origins of ergonomics using two scientometric techniques named RPYS and RPYS-Co.
\end{abstract}

Methods: This applied research was conducted in a scientometrics method. The research data consists of 31239 articles in the field of Ergonomics which were indexed over the 20-year period (2000-2019) in Web of Science database. After extracting all the references of these articles, the results were analyzed using the CRExplorer software.

Results: Results showed that in the field of ergonomics, from 1700 to 2000, a total of 19 mutations occurred in the two periods of 1900-1700 and 2000-1900. The article "the information capacity of the human motor system in controlling the amplitude of movement" by Fitts (1954) was recognized as an outstanding and index work. According to RPYS-CO results, the most co-citations with index work are related to the works of 1997 and 2004, which are on Fitts Law.

Conclusion: In the period of 1700 to1900 influential works were related to psychology, biology, and economics, and during 1900-2000 influential works were related to psychological theories, work environment, and research methodology.

Keywords: Ergonomics; Citation analysis; Scientometrics

How to Cite This Article:

Heidarimoghadam R, Khasseh A A, Vakilimofrad H, Fattahi A, Amiri M R. Identification and Analysis of the Historical Origins of Ergonomics by Referenced Publication Year's Spectroscopy. Iran J Ergon. 2021; 9(2):42-57 


\section{Extended Abstract}

\section{Introduction}

Every scientific activity needs to know the previous related scientific activities so that its various aspects can be better investigated in order to obtain a richer intellectual heritage.

Since the introduction of RPYS and RPYS-CO techniques, studies have been conducted to identify the historical origins of various fields. Historically, for the first time, Marks et al. [14] introduced the RPYS technique, using this method to conduct research on the scientific production of graphene and solar cells and to identify and analyze important works in these two fields. In the same year, Leydesdorff et al. (2014) investigated the origins of scientometrics using the RPYS method [15]. In Iran, for the first time, Soheili et al. (2015) examined the origins of the field of information behavior using the RPYS method and showed that the area of information behavior, in addition to psychology, had been partially influenced by quantitative and qualitative methodological effects (such as grounded theory). [16].
This article aims to identify and analyze the historical origins of ergonomics using two scientometric techniques named RPYS and RPYS-Co.

\section{Methods}

This applied research was conducted in a scientometrics method. The research data consists of 31239 articles in the field of Ergonomics, which were indexed over the 20-year period (2000-2019) in Web of Science database. After extracting all the references of these articles, the results were analyzed using the CRExplorer software.

\section{Results}

Results showed that in the field of ergonomics, from 1700 to 2000 , a total of 19 mutations occurred in the two periods of 1900-1700 and 2000-1900. The article "the information capacity of the human motor system in controlling the amplitude of movement" by Fitts (1954) was recognized as an outstanding and index work. According to RPYS-CO results, the most cocitations with index work are related to the works of 1997 and 2004, which are on Fitts Law.

Table 1. Historical mutations in the field of ergonomics with the most referenced works in the period 1700-1900.

\begin{tabular}{|c|c|c|c|c|}
\hline $\begin{array}{l}\text { Mutation } \\
\text { year }\end{array}$ & $\begin{array}{c}\text { Profile of the most effective document in } \\
\text { the year of the mutation }\end{array}$ & $\begin{array}{l}\text { Number of } \\
\text { citations } \\
\text { received by } \\
\text { the document }\end{array}$ & $\begin{array}{c}\text { Total } \\
\text { number of } \\
\text { citations } \\
\text { received per } \\
\text { mutation } \\
\text { year }\end{array}$ & $\begin{array}{l}\text { Document } \\
\text { type }\end{array}$ \\
\hline 1776 & $\begin{array}{l}\text { Smith, A. (1776). An inquiry into the nature } \\
\text { and causes of the wealth of nations: Volume } \\
\text { One. London: printed for W. Strahan; and T. } \\
\text { Cadell, } 1776 .\end{array}$ & 13 & 13 & Book \\
\hline 1860 & $\begin{array}{c}\text { Fechner, G. T. (1860). Elemente der } \\
\text { psychophysik (Vol. 2). Breitkopf u. Härtel. }\end{array}$ & 14 & 18 & Book \\
\hline 1872 & $\begin{array}{c}\text { Darwin, C. (1872). The expression of the } \\
\text { emotions in man and animals. London: } \\
\text { Murray. }\end{array}$ & 15 & 17 & Book \\
\hline 1890 & $\begin{array}{c}\text { James, W. (1890). The Principles of } \\
\text { Psychology, in two volumes. New York: } \\
\text { Henry Holt and Company. }\end{array}$ & 43 & 66 & Book \\
\hline 1890 & $\begin{array}{l}\text { Warren, S. D., \& Brandeis, L. D. (1890). } \\
\text { Right to privacy. Harv. L. Rev., 4, } 193 .\end{array}$ & 14 & 66 & Article \\
\hline 1899 & $\begin{array}{c}\text { Woodworth, R. S. (1899). Accuracy of } \\
\text { voluntary movement. The Psychological } \\
\text { Review: Monograph Supplements, 3(3), i. }\end{array}$ & 34 & 51 & Article \\
\hline
\end{tabular}


Table 2. Historical mutations in the field of ergonomics with the most referenced works in the period 1900-2000

\begin{tabular}{|c|c|c|c|c|}
\hline $\begin{array}{l}\text { Mutation } \\
\text { year }\end{array}$ & $\begin{array}{c}\text { Profile of the most effective document in the } \\
\text { year of the mutation }\end{array}$ & $\begin{array}{l}\text { Number of } \\
\text { citations } \\
\text { received by } \\
\text { the document }\end{array}$ & $\begin{array}{c}\text { Total } \\
\text { number of } \\
\text { citations } \\
\text { received per } \\
\text { mutation } \\
\text { year }\end{array}$ & $\begin{array}{l}\text { Document } \\
\text { type }\end{array}$ \\
\hline 1908 & $\begin{array}{l}\text { Yerkes, R.M., \& Dodson, J.D. (1908). The relation } \\
\text { of strength of stimulus to rapidity of habit- } \\
\text { formation, Journal of Comparative Neurology and } \\
\text { Psychology, vol.18, issue.5, pp.459-82. }\end{array}$ & 71 & 109 & Article \\
\hline 1911 & $\begin{array}{l}\text { Taylor, F. (1911). The Principles of Scientific } \\
\text { Management. Mineola, NY: Dover Publications. }\end{array}$ & 44 & 76 & Book \\
\hline \multirow[t]{2}{*}{1932} & $\begin{array}{l}\text { Bartlett, F. C. (1932). Remembering: A study in } \\
\text { experimental and social psychology. Cambridge } \\
\text { University Press }\end{array}$ & 52 & \multirow[t]{2}{*}{179} & Book \\
\hline & $\begin{array}{l}\text { Likert, R., 1932. A technique for the measurement } \\
\text { of attitudes. Archives of Psychology 22, 1-55. }\end{array}$ & 44 & & Article \\
\hline 1938 & $\begin{array}{l}\text { Gibson, J. J., \& Crooks, L. E. (1938). A theoretical } \\
\text { field-analysis of automobile-driving. The American } \\
\text { journal of psychology, 51(3), 453-471. }\end{array}$ & 45 & 233 & Article \\
\hline
\end{tabular}

Shannon, C. E. (1948). A mathematical theory of communication. The Bell system technical

Article

1948 journal, 27(3), 379-423.

Mackworth, N.H.(1948). The breakdown of vigilance during prolonged visual search. Quarterly Journal of Experimental Psychology, 1, 6-21.

Article

1951 Cronbach, L. J. (1951). Coefficient alpha and the internal structure of tests. Psychometrika, 16, 297-334

Article

Fitts, P. M. (1954). The information capacity of the

1954 human motor system in controlling the amplitude of movement. Journal of experimental

Article psychology, 47(6), 381.

Glaser, Barney G. and Strauss, Anselm L. (1967)

1967 The discovery of grounded theory: strategies for qualitative research. Chicago.: Aldine

$127 \quad 1864 \quad$ Book

Fishbein, M., \& Ajzen, I. (1975). Belief, Attitude,

1975 Intention, and Behavior: An Introduction to Theory and Research. Reading, MA: Addison-Wesley.

Nunnally, J. C. (1978). Psychometric Theory (2nd ed.). New York: McGraw-Hill.

Vygotsky, L. S. (1978). Mind in society: The development of higher psychological processes Cambridge, Mass.: Harvard University Press

Snook, S. H. (1978). The design of manual handling tasks. Ergonomics, 21(12):963-85

1980
Zohar, D. (1980) Safety climate in industrial organizations Theoretical and applied implications. The Journal of Applied Psychology, 65, 96-102
280
101

4287

99

Article

Book
Book

175 


\begin{tabular}{|c|c|c|c|c|}
\hline $\begin{array}{l}\text { Mutation } \\
\text { year }\end{array}$ & $\begin{array}{c}\text { Profile of the most effective document in the } \\
\text { year of the mutation }\end{array}$ & $\begin{array}{l}\text { Number of } \\
\text { citations } \\
\text { received by } \\
\text { the document }\end{array}$ & $\begin{array}{c}\text { Total } \\
\text { number of } \\
\text { citations } \\
\text { received per } \\
\text { mutation } \\
\text { year }\end{array}$ & $\begin{array}{l}\text { Document } \\
\text { type }\end{array}$ \\
\hline \multirow{3}{*}{1988} & $\begin{array}{l}\text { Ajzen, I. \& Fishbein, M. (1980). Understanding } \\
\text { attitudes and predicting social behavior. Englewood } \\
\text { Cliffs: Prentice-Hall }\end{array}$ & 149 & \multirow{3}{*}{12998} & Book \\
\hline & $\begin{array}{l}\text { Hart, S. G., \& Staveland, L. E. (1988). } \\
\text { Development of NASA-TLX (Task Load Index): } \\
\text { Results of empirical and theoretical research. } \\
\text { In Advances in psychology (Vol. 52, pp. 139-183). } \\
\text { North-Holland. }\end{array}$ & 596 & & Article \\
\hline & $\begin{array}{l}\text { Cohen, J. (1988). Statistical Power Analysis for the } \\
\text { Behavioral Sciences (2nd ed.). Hillsdale, NJ: } \\
\text { Lawrence Erlbaum. }\end{array}$ & 411 & & Book \\
\hline \multirow[b]{2}{*}{1993} & $\begin{array}{l}\text { Nielsen, J. (1993). Usability engineering. Morgan } \\
\text { Kaufmann. }\end{array}$ & 493 & \multirow[b]{2}{*}{21576} & Book \\
\hline & $\begin{array}{l}\text { Waters, T. R., Putz-Anderson, V., Garg, A., \& Fine, } \\
\text { L. J. (1993). Revised NIOSH equation for the } \\
\text { design and evaluation of manual lifting } \\
\text { tasks. Ergonomics, 36(7), 749-776. }\end{array}$ & 319 & & Article \\
\hline 1995 & $\begin{array}{l}\text { Endsley, M.R. (1995) Toward a Theory of Situation } \\
\text { Awareness in Dynamic Systems. Human Factors: } \\
\text { The Journal of the Human Factors and Ergonomics } \\
\text { Society, 37, 32-64. }\end{array}$ & 589 & 27155 & Article \\
\hline
\end{tabular}

\section{Discussion}

According to the research results, a total of 19 mutations have occurred in the evolutionary process of ergonomics, of which five mutations occurred in the period 1900-1700. The most important mutation of this period took place in 1890 , when it was related to two works, the book by James [20] and the article by Warren [21]. These two works, with the number of citations they had in 1890, caused a major mutation in this field. The second major mutation in this period occurred in 1899 after the publication of Woodworth's work. Other mutations in this period include Smith's book in 1776 [23], Fechner's in 1860 [24], and "The expression of the Emotions in Man and Animals" is a work by Darwin in 1872 [25].

During 2000-1900, fourteen mutations were identified, which are related to the publication of 9 books and 12 articles; these are the most influential works published in the 1980s and 1990s. The most influential mutation forward was in 1995 after the publication of Endsley's paper "Toward a theory of situation awareness in dynamic systems" [26]. The second major mutation in this period occurred in 1993 with Nielsen [27] and Waters et al. [28].

\section{Conclusion}

In the period of 1700 to1900 influential works were related to psychology, biology, and economics, and during 1900-2000 influential works were related to psychological theories, work environment, and research methodology.

\section{Acknowledgement}

The authors of this article would like to thank the Vice Chancellor for Research and Technology of Hamadan University of Medical Sciences for approving the research plan and cooperating in its implementation.

\section{Conflict of Interest}

The authors declared no conflict of interest. 


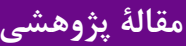

شناسايى و تحليل خاستخاههاى تاريخى حوزة اركونومى به روش طيفسنجى سال انتشار منابع

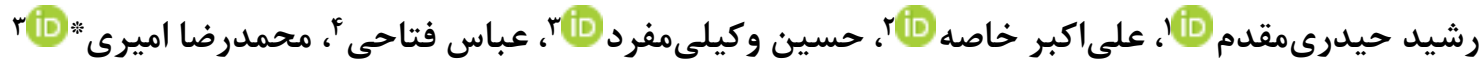

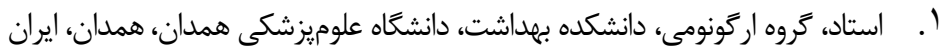

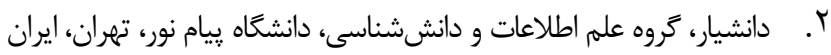

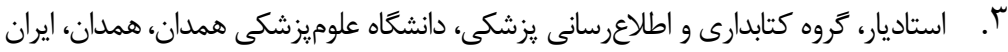

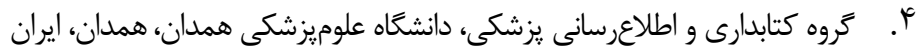

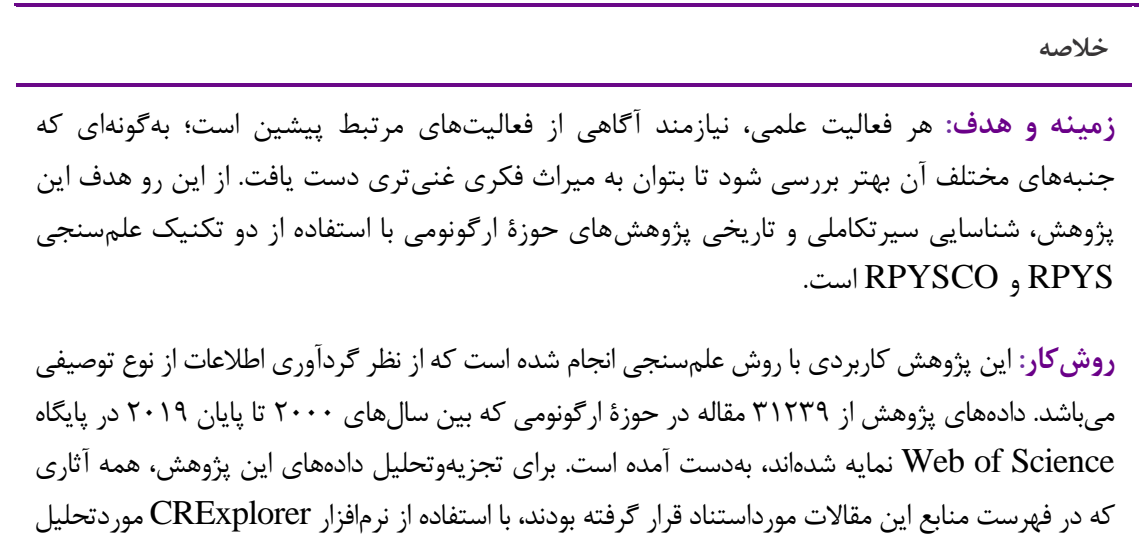
قرار كرفت.

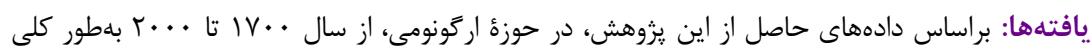

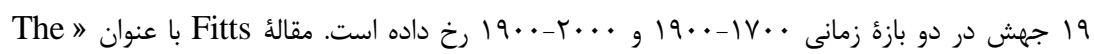
Information Capacity Of The Human Motor System In Controlling The Amplitude Of Movement

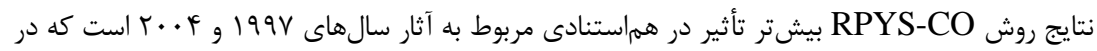

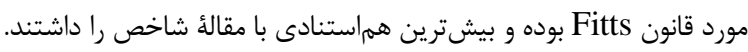

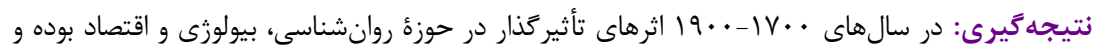

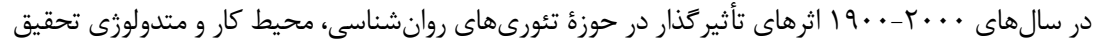

بودند.

كليدوازهها: اركونومى، تحليل استنادى، علمسنجى

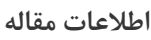

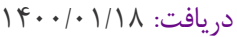

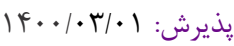

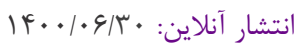

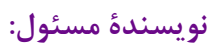

محمدرضا اميرى

كروه كتابدارى و اطلاعرسانى يزشكى

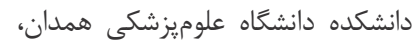

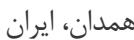

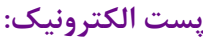

m.r.amirilib@gmail.com

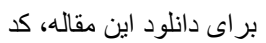

زير را با موبايل خود اسكن مئن كنبد

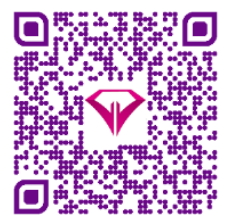

كبىرايت C) مجله اركونومى؛ دسترسى آزاد؛

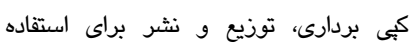
غيرتجارى با ذكر منبع آزاد است.

مقل مه

آنها تحميل مىكند، منطبق و متناسب سازند و با

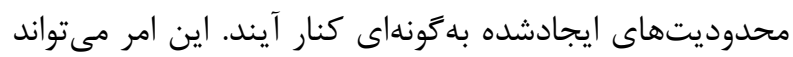

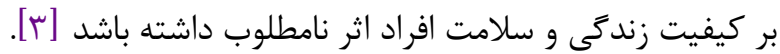
علوم روز جهان بخش عمدهاى از مشكلات افراد در

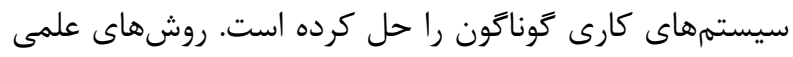

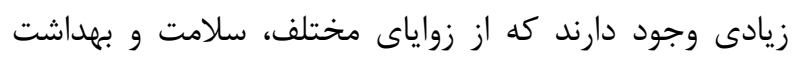
انسانها و نيز كار ايى بهعنوان بخشى از راه راه رسيدن به بهرئورى
نيروى كار يكى از عناصر مهمم و كليدى در توسعهُ هر جامعه

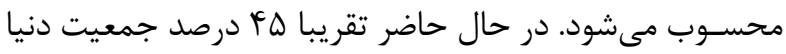

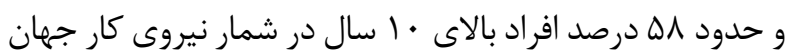
محسوب مىشوند. از جمله موضوعات بسيار مهرم در ارتباط با دال

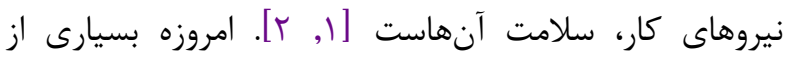

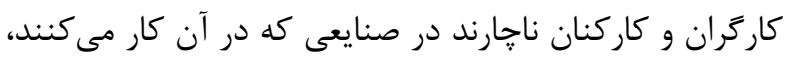
خود را با شرايط نامناسبى كه محيط و ابزار مورد استفاده بر 
سؤال كه جه موضوعاتى در گذشته و آينده مطرح بوده و خواهند

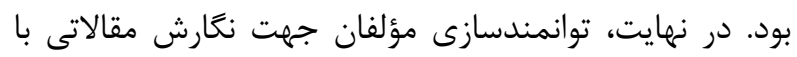
بيشترين استناد [9].

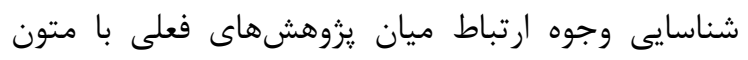
كذشته، نقش بسزايى در توسعهُ علم ايفا مى كند. در واقع علم

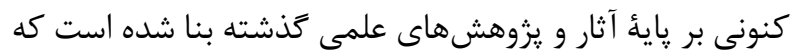

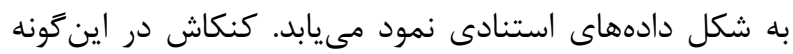

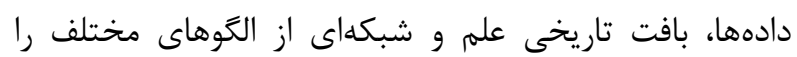

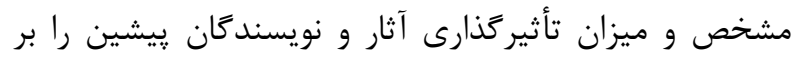

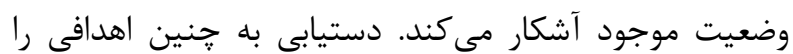

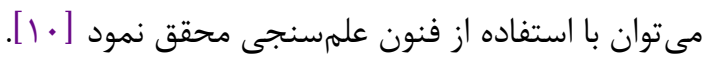

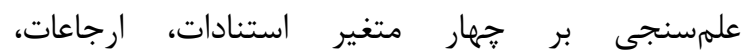
يديدآورندًان و انتشارات علمى استوار است. بر همين اساس

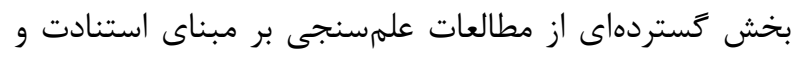

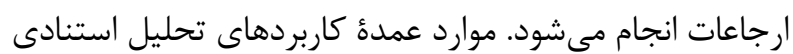

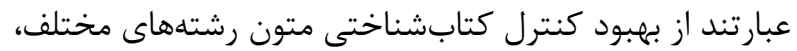

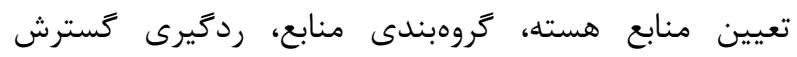

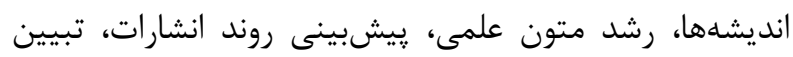

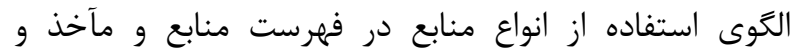

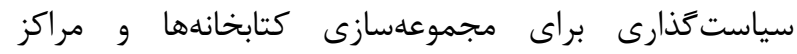
اطلاعرسانى. از جمله موارد مهمى كه بـ با استفاده از مطالعات

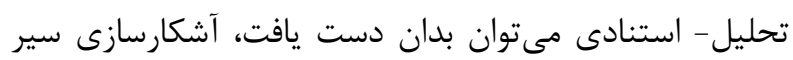
تحول تاريخى حوزههاى يزوهش بر براس اساس سال انتشار است

روش طيفسنجى سال انتشار مآخذ كه توسط Bornmann

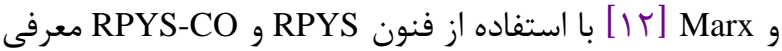

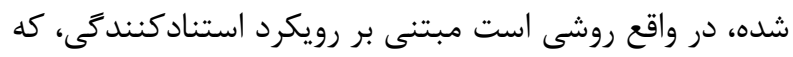
در آن، فهرست منابع استناد شده با تأكيد بر سال انتشار آنها

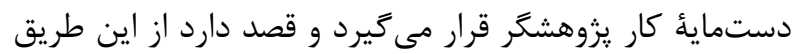
نشان دهد كه جه آثار و نويسندكانى در شكل

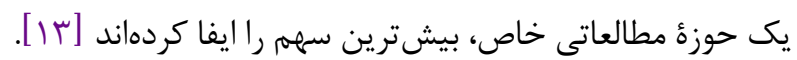

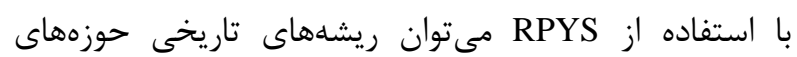

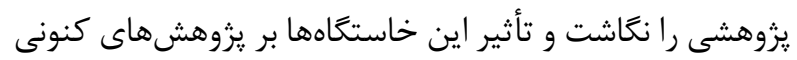

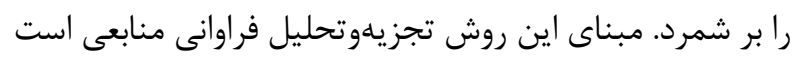

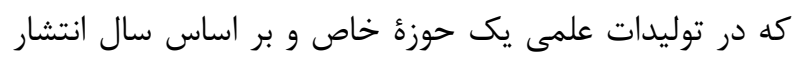

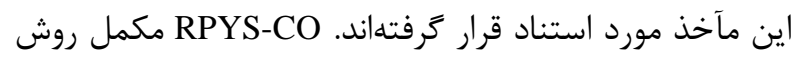

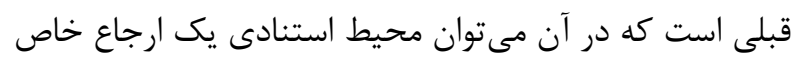

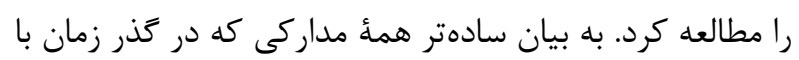

را مورد تجزيهوتحليل قرار مىدهند. يكى از اين علوم اركَونومى إنى

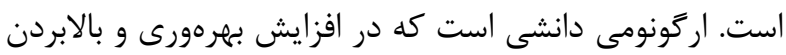
سطح تندرستى كاركنان به يارى انسان مىشتابد، رابطئ متقابل

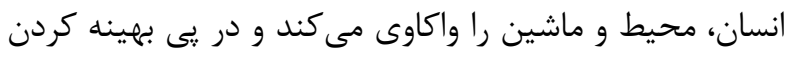

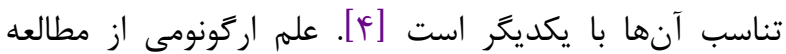

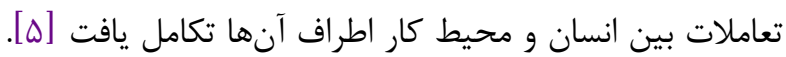

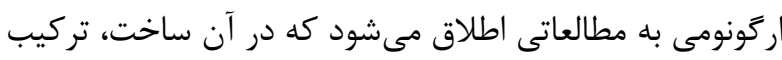

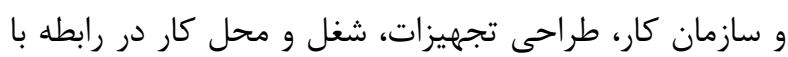

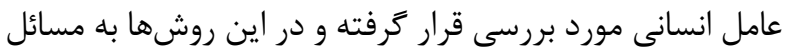

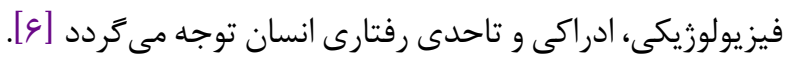

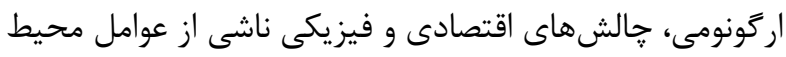

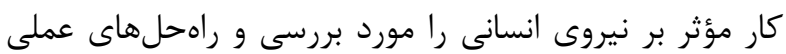

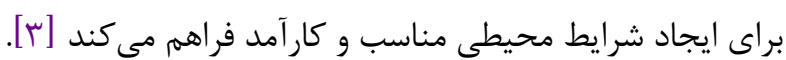

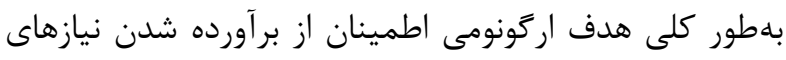

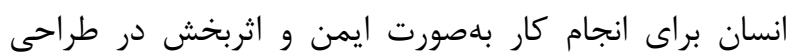
سيستمهاى كارى است. اين علم و حرفه در دوران پِ از جنَّجهانى دوم ايجاد

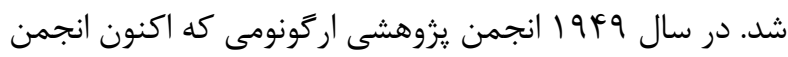

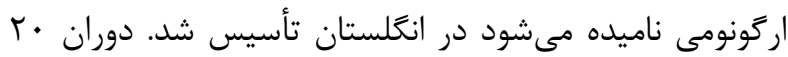

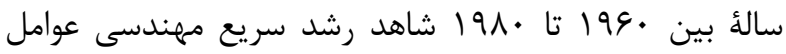

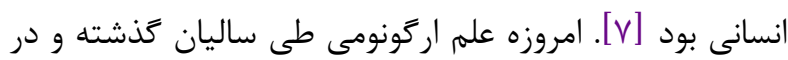
خلال تحقيقات گسترده كه در اين حوزه انجام شده، اهميت آن آن را در كاهش مشكلات جسمانى مثل ناراحتىهاى عضلانىـ

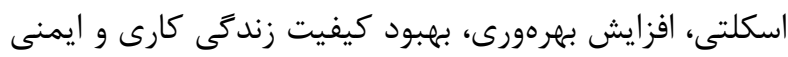

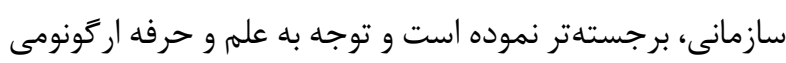

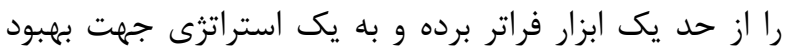

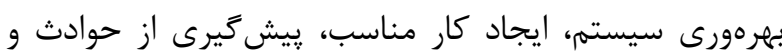
بيمارى هاى ناشى از كار و بهبود راندمان و عملكرد انسان تبديل برديل

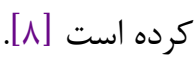

در هر حوزء علمى، فعاليتهاى يزوهشى براساس

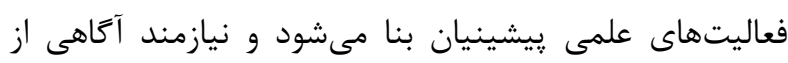

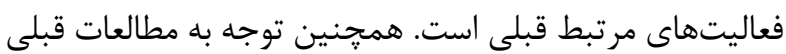
و جايغاه آنها مىتواند اهميت زيادى داشته باشد. نخست،

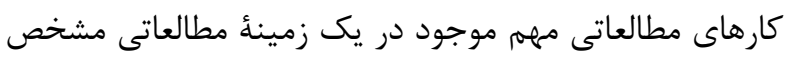

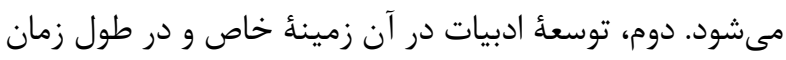
كه مىتواند در تعيين موضوعهاى محورى و شناسايى افراد كليدى در يك زمينهُ مطالعاتى يارىرسان باشد. سوم، ترسيم

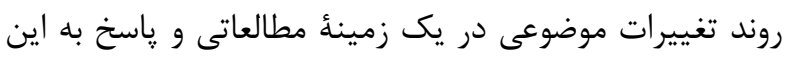




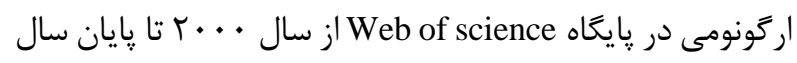

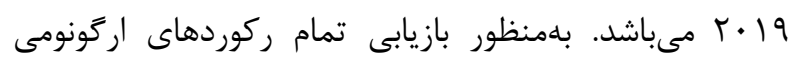

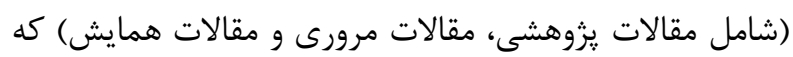

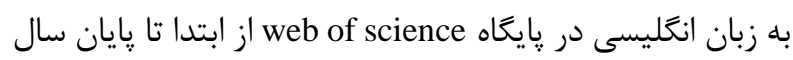

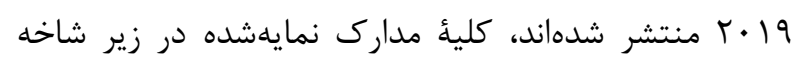
ergonomics

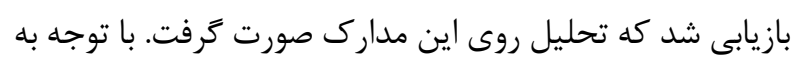

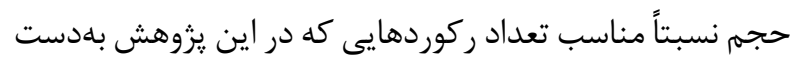

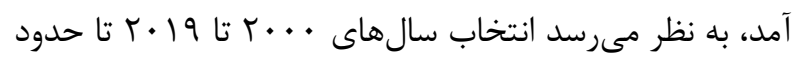

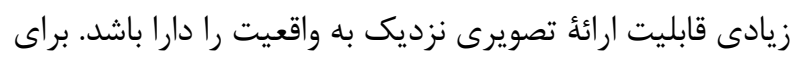

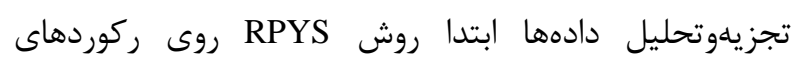

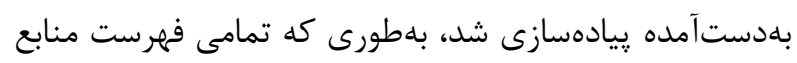

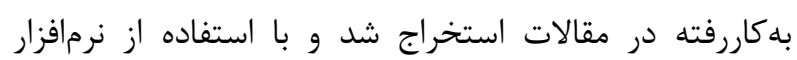
CRExplorer

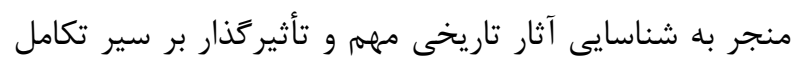

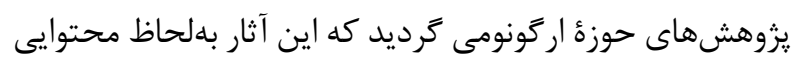

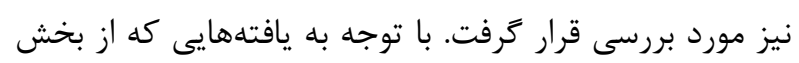

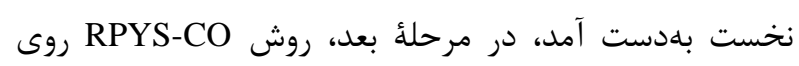

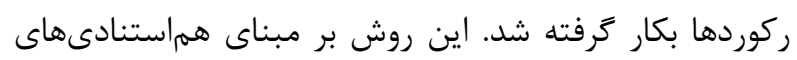

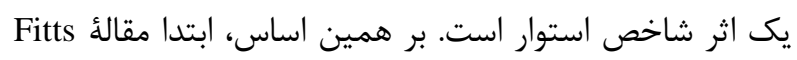

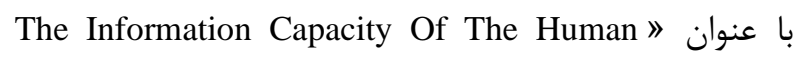
Motor System In Controlling The Amplitude Of "Movement

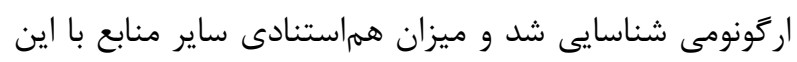

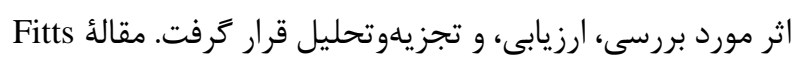

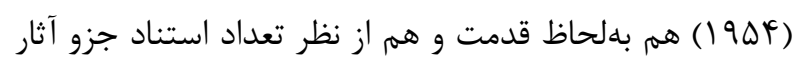

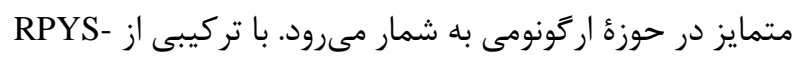

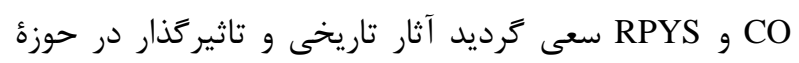

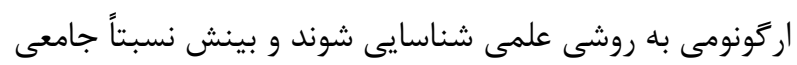
از سير تكاملى اين حوزه بلهدست آيد.

\section{يافته ها}

يافتهاى اين يزوهش در دو بخش كلى RPYS و -RPS

$$
\text { CO }
$$

\section{نتايج RPYS}

در اين قسمت تجزيدوتحليل يافتههاى حاصل از تكنيكهاى RPYS

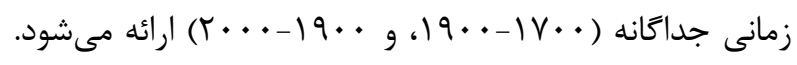

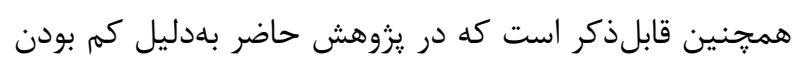

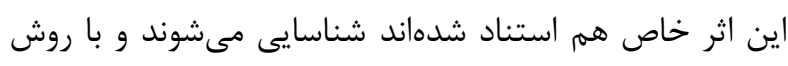

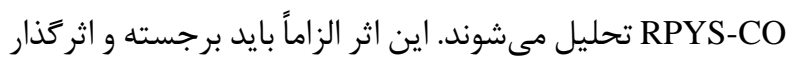

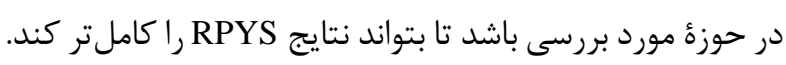

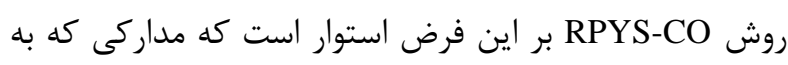

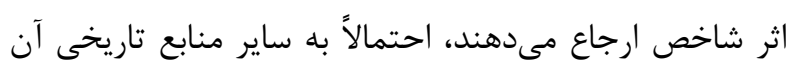
حوزه استناد مى دهند [11]

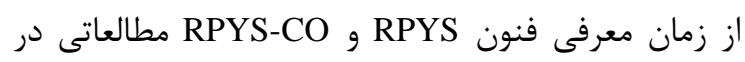

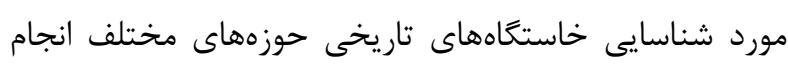

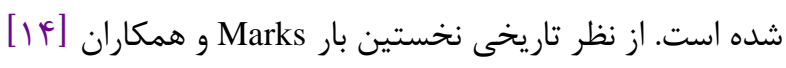

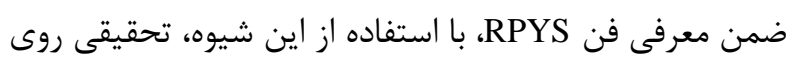

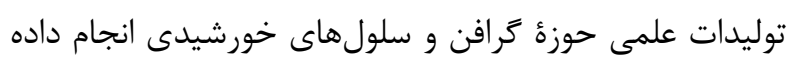

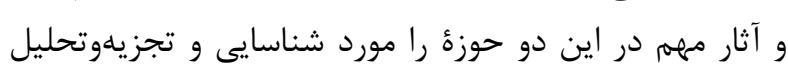

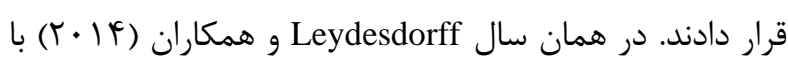
روش RPYS خاستكاههاى حوزء علمسنجى را بررسى كردند

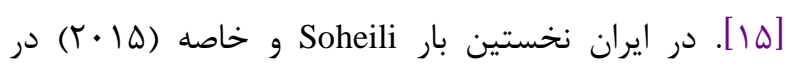

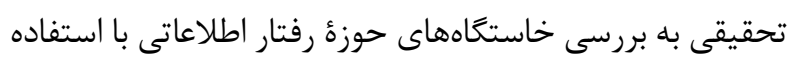

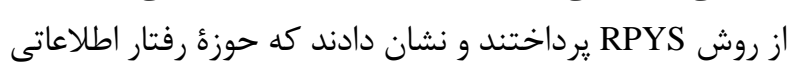

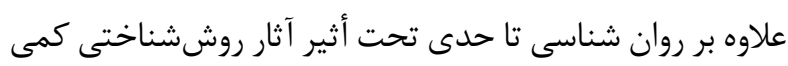

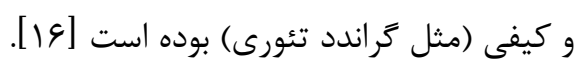

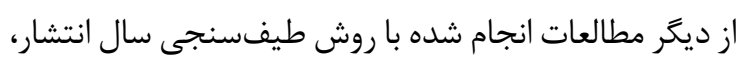

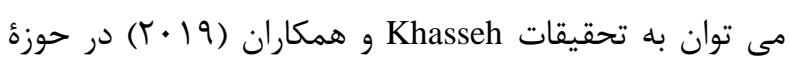

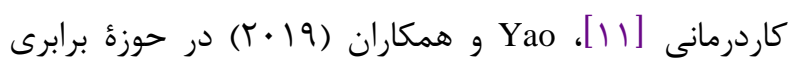

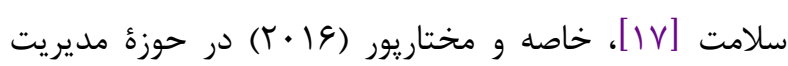

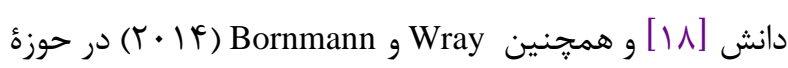
فلسفهُ علم [19 1 ] اشاره كرد.

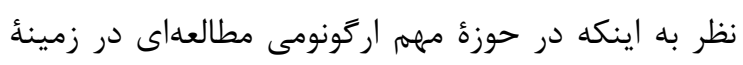

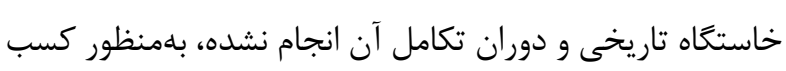

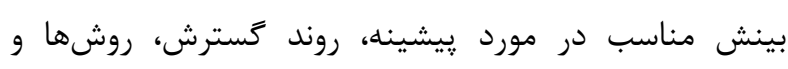

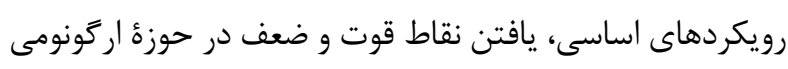

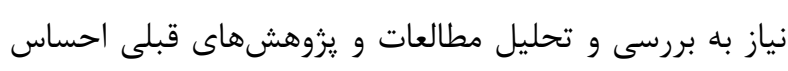

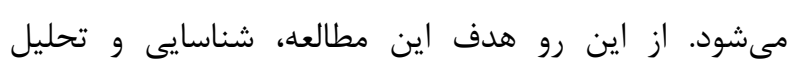

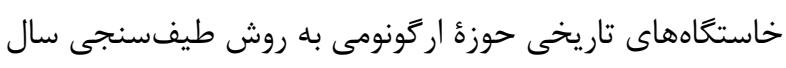

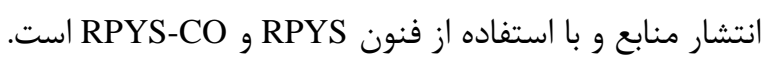

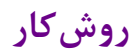

اين يزوهش كاربردى با استفاده از روشهاى رايج در

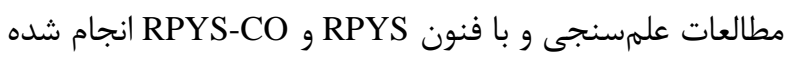

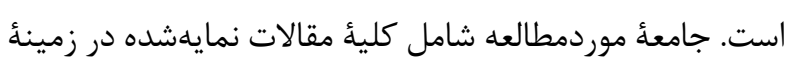


مهمى در شكل گيرى مباحث ارگونومى روى داده است. بهعبارتى اين آثار تأثيركذارى بيشترى ديرى در اين حوزه داشتهاند

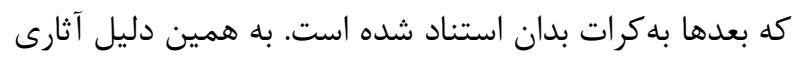
كه در اين سالها جاب شدهاند، موردبحث قرار ميى گيرند تا تأثير

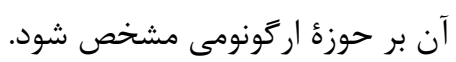

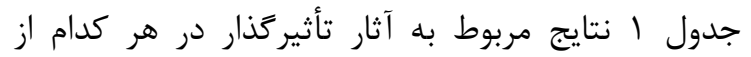

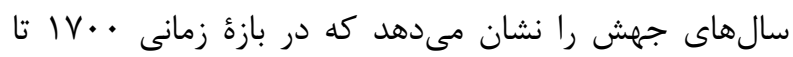

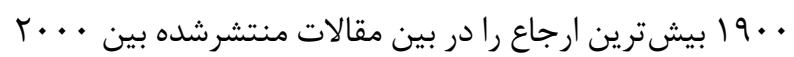

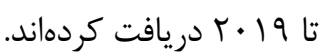

تعداد استنادات قبل از بازهُ زمانى . . . . مطالعات بعد از اين سال را در نظر گرفته است.

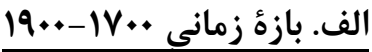

شكل I (الف). توزيع تعداد مآخذ موجود در ركوردهاى حوزه

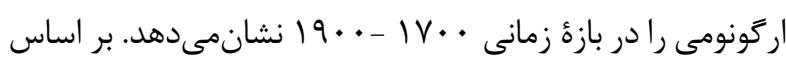

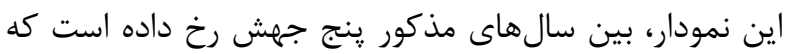

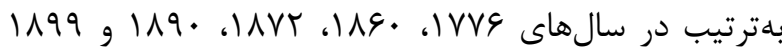
روى داده است. بنابراين، بهاحتمال فراوان در اين سالها، رخداد

\begin{tabular}{|c|c|c|c|c|}
\hline نوع اثر & 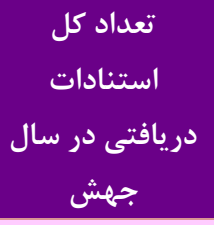 & تعداد استنادات & مشخصات تاثير كذارترين اثر در سال جهش & سال جهش \\
\hline كتاب & Ir & Ir & $\begin{array}{l}\text { Smith, A. (1776). An inquiry into the nature and causes } \\
\text { of the wealth of nations: Volume One. London: printed } \\
\text { for W. Strahan; and T. Cadell, } 1776 .\end{array}$ & IVVS \\
\hline كتاب & 11 & If & $\begin{array}{l}\text { Fechner, G. T. (1860). Elemente der psychophysik } \\
\text { (Vol. 2). Breitkopf u. Härtel. }\end{array}$ & 1Aq. \\
\hline كتاب & IV & 10 & $\begin{array}{l}\text { Darwin, C. (1872). The expression of the emotions in } \\
\text { man and animals. London: Murray. }\end{array}$ & IAVT \\
\hline كتاب & 49 & er & $\begin{array}{l}\text { James, W. (1890). The Principles of Psychology, in two } \\
\text { volumes. New York: Henry Holt and Company. }\end{array}$ & $1 \wedge 9$. \\
\hline مقاله & 99 & If & $\begin{array}{c}\text { Warren, S. D., \& Brandeis, L. D. (1890). Right to } \\
\text { privacy. Harv. L. Rev., 4, } 193 .\end{array}$ & 119. \\
\hline مقاله & QI & MF & $\begin{array}{c}\text { Woodworth, R. S. (1899). Accuracy of voluntary } \\
\text { movement. The Psychological Review: Monograph } \\
\text { Supplements, 3(3), i. }\end{array}$ & 1199 \\
\hline
\end{tabular}

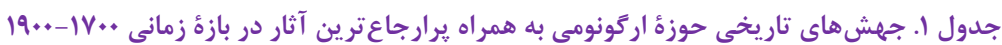

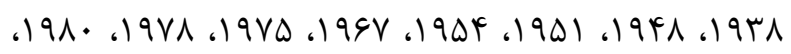

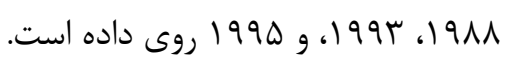

همجنين جدول r نتايج مربوط به آثار تاثير گذار در هر كدام

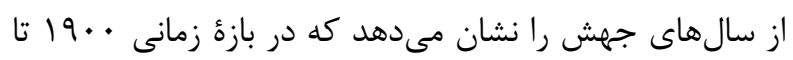

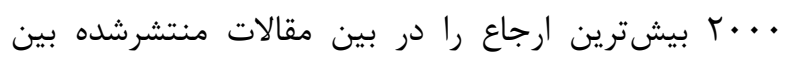

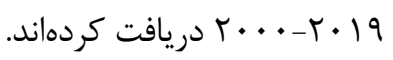

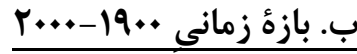

شكل ا (ب) توزيع تعداد مآخذ موجود در ركوردهاى حوزه

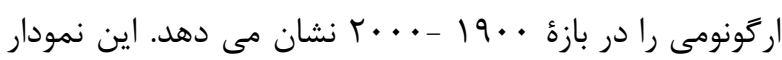

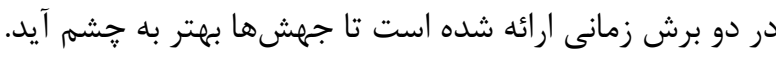

\begin{tabular}{|c|c|c|c|c|}
\hline 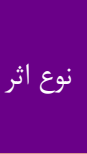 & 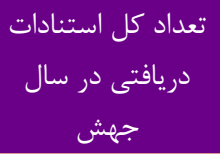 & 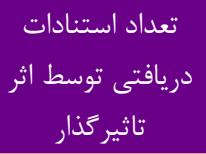 & مشخصات تاثير گذارترين اثر در سال جهش & سال \\
\hline مقاله & 1.9 & VI & $\begin{array}{c}\text { Yerkes, R.M., \& Dodson, J.D. (1908). The relation of } \\
\text { strength of stimulus to rapidity of habit-formation, Journal } \\
\text { of Comparative Neurology and Psychology, vol.18, issue.5, } \\
\text { pp.459-82. }\end{array}$ & $19 \cdot 1$ \\
\hline كتاب & VG & FY & $\begin{array}{c}\text { Taylor, F. (1911). The Principles of Scientific Management. } \\
\text { Mineola, NY: Dover Publications. }\end{array}$ & 1911 \\
\hline
\end{tabular}

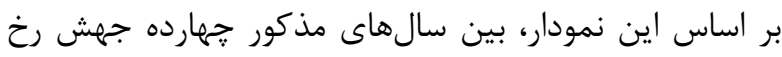

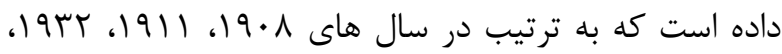

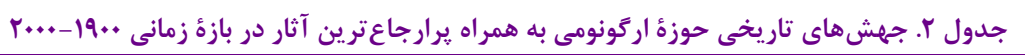




\begin{tabular}{|c|c|c|c|c|}
\hline نوع اثر & تعداد كل استنادات & 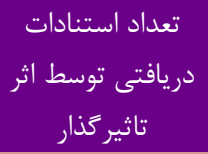 & مشخصات تاثير كذارترين اثر در سال جهش & جهش \\
\hline كتاب & 189 & $\Delta T$ & $\begin{array}{l}\text { Bartlett, F. C. (1932). Remembering: A study in } \\
\text { experimental and social psychology. Cambridge University } \\
\text { Press } \\
\text { Likert, R., 1932. A technique for the measurement of } \\
\text { attitudes. Archives of Psychology 22, 1-55. }\end{array}$ & 19rT \\
\hline مقاله & זrז & is & $\begin{array}{c}\text { Gibson, J. J., \& Crooks, L. E. (1938). A theoretical field- } \\
\text { analysis of automobile-driving. The American journal of } \\
\text { psychology, 51(3), 453-471. }\end{array}$ & $194 \wedge$ \\
\hline مقاله & \multirow{2}{*}{ FrT } & 41 & $\begin{array}{l}\text { Shannon, C. E. (1948). A mathematical theory of } \\
\text { communication. The Bell system technical journal, 27(3), } \\
\text { 379-423. }\end{array}$ & \multirow[t]{2}{*}{$194 \wedge$} \\
\hline مقاله & & Fr & $\begin{array}{c}\text { Mackworth, N.H.(1948). The breakdown of vigilance during } \\
\text { prolonged visual search. Quarterly Journal of Experimental } \\
\text { Psychology, 1, 6-21. }\end{array}$ & \\
\hline مقاله & $\Delta T_{q}$ & $11 \mathrm{~V}$ & $\begin{array}{l}\text { Cronbach, L. J. (1951). Coefficient alpha and the internal } \\
\text { structure of tests. Psychometrika, 16, 297-334 }\end{array}$ & 1901 \\
\hline مقاله & $\wedge \varepsilon$. & TTA & $\begin{array}{l}\text { Fitts, P. M. (1954). The information capacity of the human } \\
\text { motor system in controlling the amplitude of } \\
\text { movement. Journal of experimental psychology, } 47(6), 381 \text {. }\end{array}$ & $19 \Delta F$ \\
\hline كتاب & INGF & ITV & $\begin{array}{c}\text { Glaser, Barney G. and Strauss, Anselm L. (1967) The } \\
\text { discovery of grounded theory: strategies for qualitative } \\
\text { research. Chicago.: Aldine }\end{array}$ & $198 \mathrm{~V}$ \\
\hline كتاب & זrTr & TMF & $\begin{array}{l}\text { Fishbein, M., \& Ajzen, I. (1975). Belief, Attitude, Intention, } \\
\text { and Behavior: An Introduction to Theory and Research. } \\
\text { Reading, MA: Addison-Wesley. }\end{array}$ & $19 \vee \Delta$ \\
\hline كتاب & \multirow{3}{*}{ FrAV } & rA. & $\begin{array}{c}\text { Nunnally, J. C. (1978). Psychometric Theory (2nd ed.). New } \\
\text { York: McGraw-Hill. }\end{array}$ & \multirow{3}{*}{$19 \vee \wedge$} \\
\hline كتاب & & $1 \cdot 1$ & $\begin{array}{c}\text { Vygotsky, L. S. (1978). Mind in society: The development } \\
\text { of higher psychological processes Cambridge, Mass.: } \\
\text { Harvard University Press }\end{array}$ & \\
\hline مقاله & & 99 & $\begin{array}{l}\text { Snook, S. H. (1978). The design of manual handling tasks. } \\
\text { Ergonomics, 21(12):963-85 }\end{array}$ & \\
\hline مقاله & \multirow{2}{*}{$\Delta \varphi \Delta F$} & $I V D$ & $\begin{array}{l}\text { Zohar, D. (1980) Safety climate in industrial organizations } \\
\text { Theoretical and applied implications. The Journal of Applied } \\
\text { Psychology, 65, 96-102 }\end{array}$ & \multirow{2}{*}{$19 \Lambda}$. \\
\hline كتاب & & 149 & $\begin{array}{c}\text { Ajzen, I. \& Fishbein, M. (1980). Understanding attitudes } \\
\text { and predicting social behavior. Englewood Cliffs: Prentice- } \\
\text { Hall }\end{array}$ & \\
\hline مقاله & \multirow[t]{2}{*}{ Ir৭१^ } & Q99 & $\begin{array}{l}\text { Hart, S. G., \& Staveland, L. E. (1988). Development of } \\
\text { NASA-TLX (Task Load Index): Results of empirical and } \\
\text { theoretical research. In Advances in psychology (Vol. 52, pp. } \\
\text { 139-183). North-Holland. }\end{array}$ & \multirow[t]{2}{*}{1911} \\
\hline كتاب & & fil & $\begin{array}{c}\text { Cohen, J. (1988). Statistical Power Analysis for the } \\
\text { Behavioral Sciences (2nd ed.). Hillsdale, NJ: Lawrence } \\
\text { Erlbaum. }\end{array}$ & \\
\hline كتاب & \multirow[b]{2}{*}{ rIQVE } & pqr & $\begin{array}{l}\text { Nielsen, J. (1993). Usability engineering. Morgan } \\
\text { Kaufmann. }\end{array}$ & \multirow[b]{2}{*}{1994} \\
\hline مقاله & & r19 & $\begin{array}{c}\text { Waters, T. R., Putz-Anderson, V., Garg, A., \& Fine, L. J. } \\
\text { (1993). Revised NIOSH equation for the design and } \\
\text { evaluation of manual lifting tasks. Ergonomics, 36(7), 749- } \\
776 .\end{array}$ & \\
\hline مقاله & TVIDD & $\Delta \wedge 9$ & $\begin{array}{c}\text { Endsley, M.R. (1995) Toward a Theory of Situation } \\
\text { Awareness in Dynamic Systems. Human Factors: The } \\
\text { Journal of the Human Factors and Ergonomics Society, 37, } \\
\text { 32-64. }\end{array}$ & 1990 \\
\hline
\end{tabular}



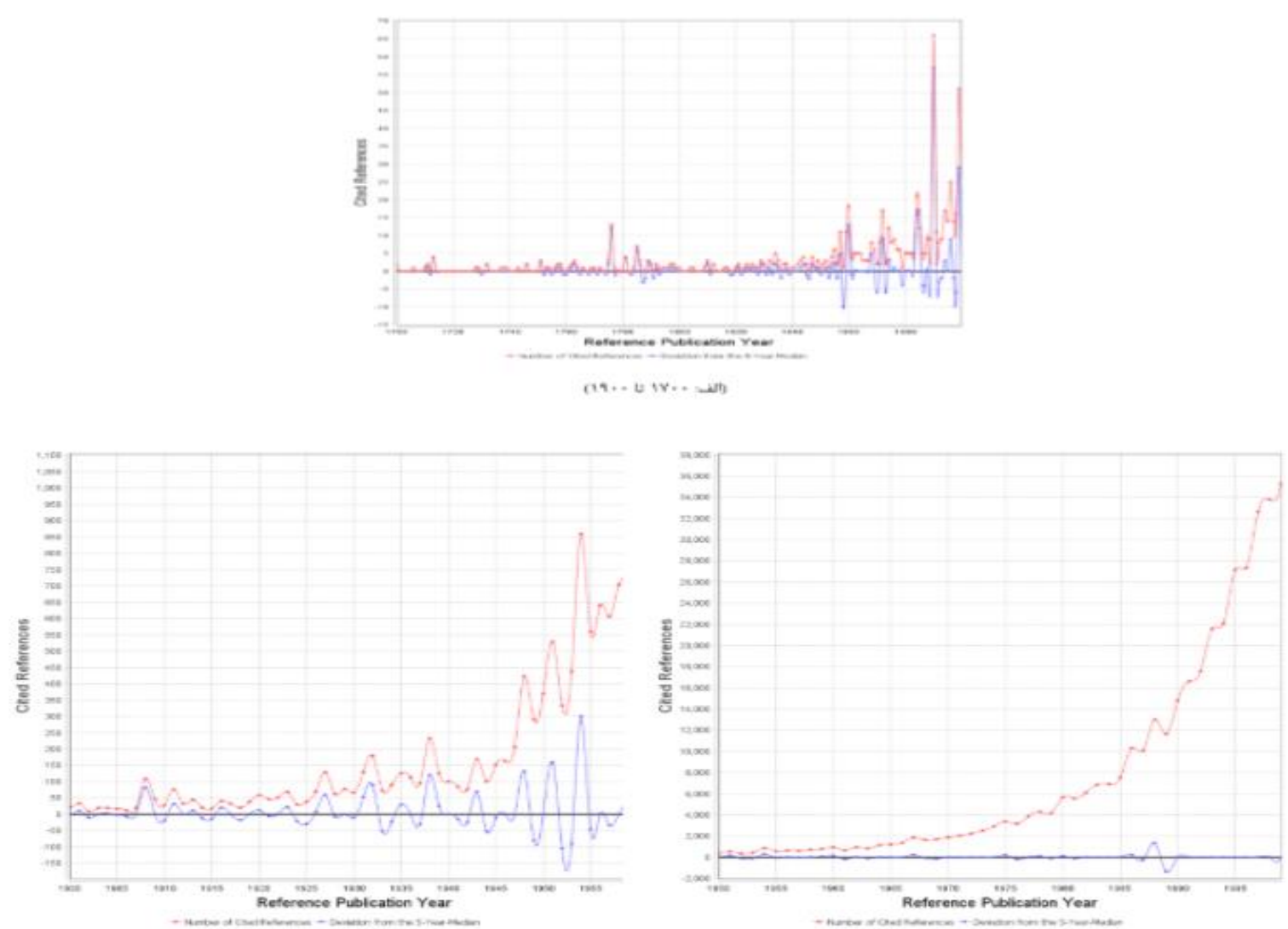

شكل ا. نتايج RPYS در موضوع اركونومى از ... تا تا ....

نتايج RPYS-CO

\section{الف. بازهٔ زمانى قبل از 19QfF}

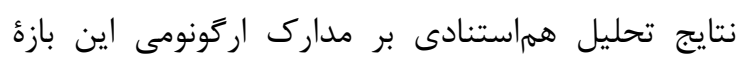

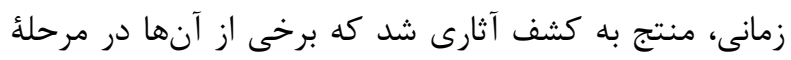

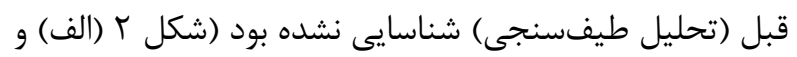

$$
\text { جدول ب). }
$$

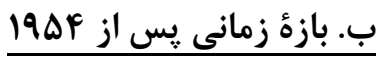

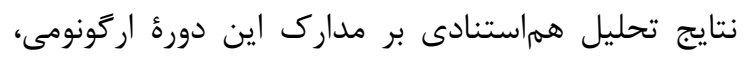

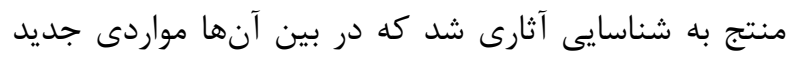

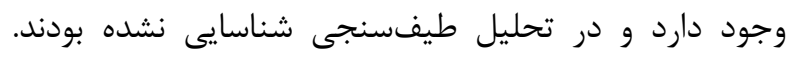

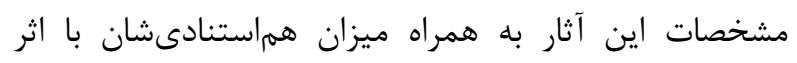
شاخص در جدول أ ارائه شده است.
در اين روش، ساير مداركى كه با اثر شاخص هماستناد

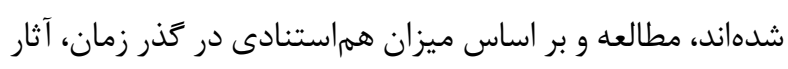

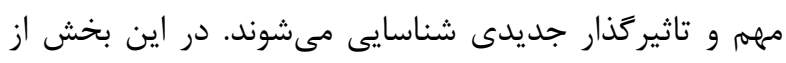

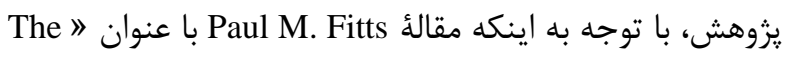
Information Capacity Of The Human Motor System In 《Controlling The Amplitude Of Movement 19DF

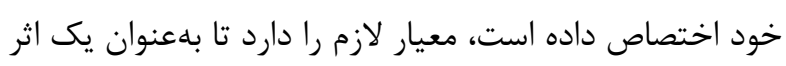

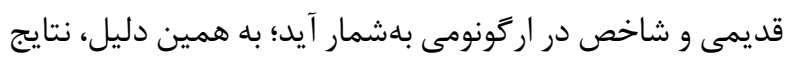

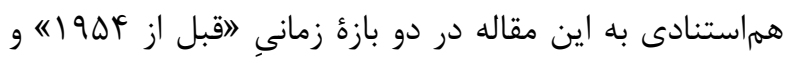

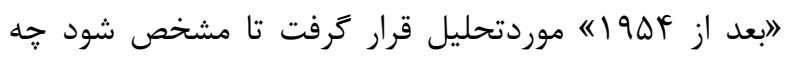

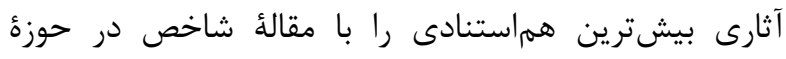

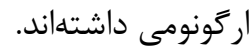




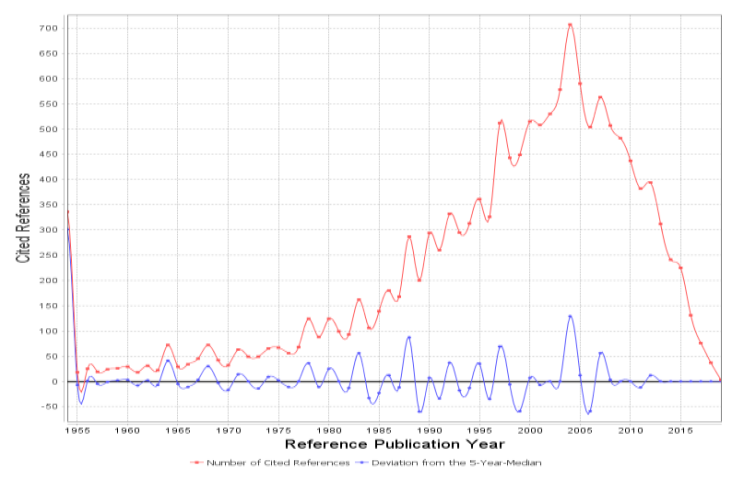

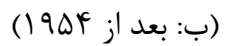

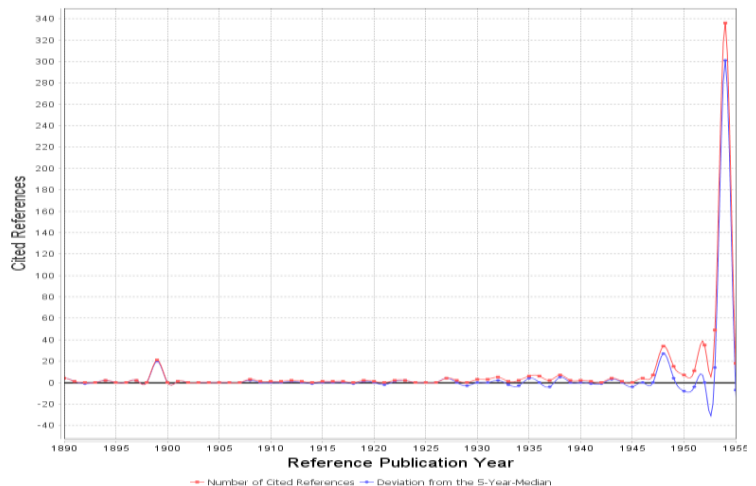

(الف: قبل از (19DF)

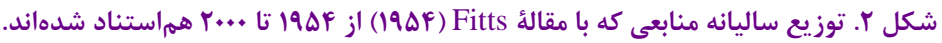

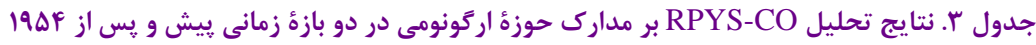

\begin{tabular}{|c|c|c|c|c|}
\hline 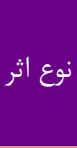 & استنادىها در كل هم & تعداد هم استنادى با & مشخصات تاثير زذارترين اثر در سال جهش & سال جهش \\
\hline \multicolumn{5}{|c|}{ بازه زمانى قبل از IOAF } \\
\hline مقاله & YI & rI & $\begin{array}{l}\text { Woodworth, R. S. (1899). The accuracy of voluntary } \\
\text { movement. Psychological Review, 3, 1-119. }\end{array}$ & 11999 \\
\hline مقاله & $r \Delta$ & rᄉ & $\begin{array}{c}\text { Hick, W. E. (1952). On the rate of gain of } \\
\text { information. Quarterly Journal of experimental } \\
\text { psychology, 4(1), 11-26. }\end{array}$ & 19Dr \\
\hline
\end{tabular}

Hyman, R. (1953). Stimulus information as a

r S S determinant of reaction time. Journal of experimental psychology, 45(3), 188.

$\begin{array}{lll}4 q & 11 & \\ & & \end{array}$

Fitts, P. M., \& Seeger, C. M. (1953). SR compatibility: spatial characteristics of stimulus and response codes. Journal of experimental psychology, 46(3), 199.

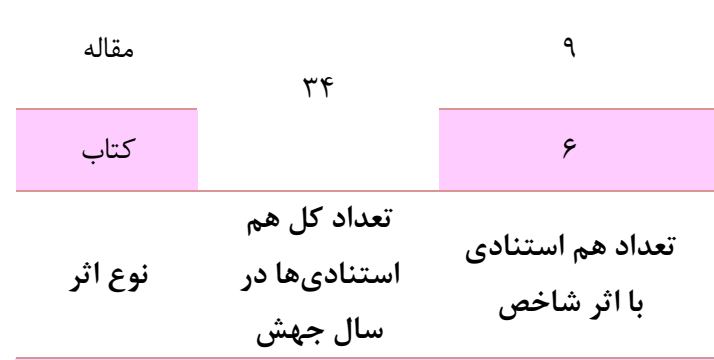

بازه زمانى يس از $19 \Delta F$

Fitts, P. M., \& Peterson, J. R. (1964). Information

\begin{tabular}{lll} 
Ner & rr \\
\hline مقاله & Rr & rr \\
\hline
\end{tabular}

مقاله $\quad$ DT

كتاب

194 capacity of discrete motor responses. Journal of experimental psychology, 67(2), 103.

A. T. Welford, A. T. (1968). Fundamentals of Skill. Methuen, London.

Card, S. K., English, W. K., \& Burr, B. J. (1978).

Evaluation of mouse, rate-controlled isometric joystick, step keys, and text keys for text selection on a CRT. Ergonomics, 21(8), 601-613.

Card, S.K., Moran, T.P. and Newell, A. (1983) The

Psychology of Human-Computer Interaction.
Shannon, C. E. (1948). A mathematical theory of communication. The Bell system technical journal, 27(3), 379-423.

Maynard, H. B., Stegemerten, G. J., \& Schwab, J. L.

$19 \% \wedge$ (1948). Methods-time measurement. McGraw-Hill

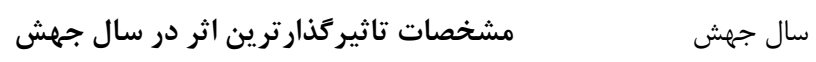

1914 Erlbaum, Hillsdale 


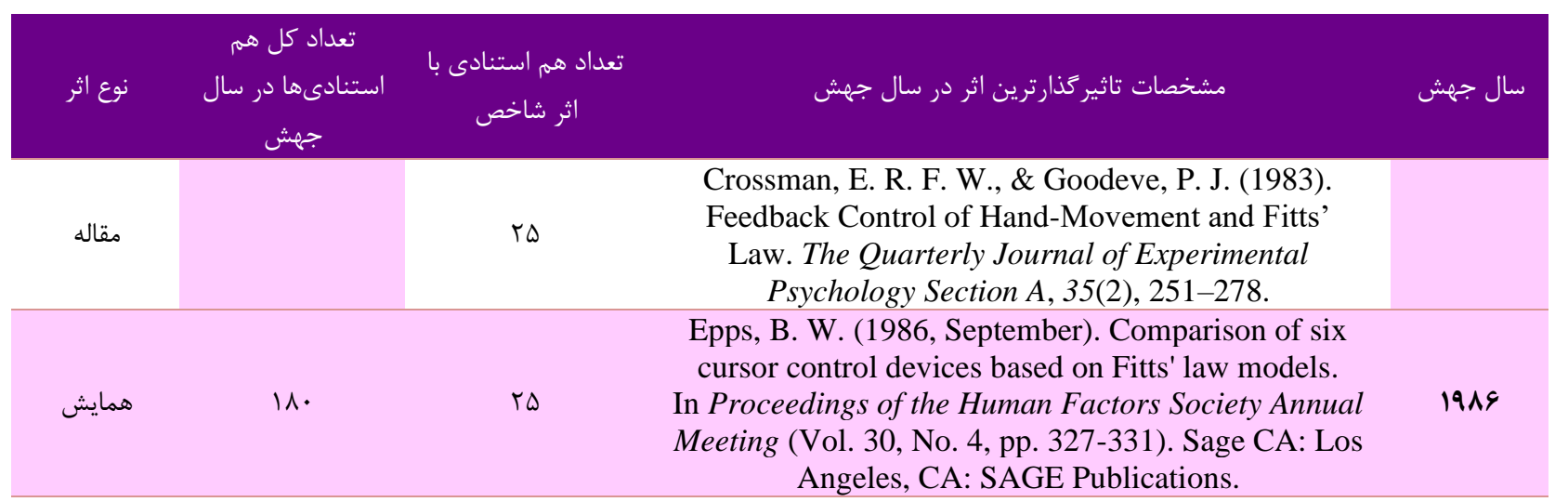

\begin{tabular}{|c|c|c|c|c|}
\hline مقاله & & r & $\begin{array}{l}\text { Gan, K. C., \& Hoffmann, E. R. (1988). Geometrical } \\
\text { conditions for ballistic and visually controlled } \\
\text { movements. Ergonomics, 31(5), 829-839. }\end{array}$ & \\
\hline مقاله & rAV & $r \Delta$ & $\begin{array}{l}\text { Meyer, D. E., Abrams, R. A., Kornblum, S., Wright, } \\
\text { C. E., \& Keith Smith, J. E. (1988). Optimality in } \\
\text { human motor performance: Ideal control of rapid } \\
\text { aimed movements. Psychological Review, 95(3), 340- } \\
370\end{array}$ & 1911 \\
\hline مقاله & Tru & 91 & $\begin{array}{l}\text { MacKenzie, I. S. (1992). Fitts' law as a research and } \\
\text { design tool in human-computer interaction. Human- } \\
\text { computer interaction, 7(1), 91-139. }\end{array}$ & 1994 \\
\hline همايش & $\Delta I T$ & $r$. & $\begin{array}{c}\text { Accot, J., \& Zhai, S. (1997, March). Beyond Fitts' law: } \\
\text { models for trajectory-based HCI tasks. In Proceedings } \\
\text { of the ACM SIGCHI Conference on Human factors in } \\
\text { computing systems (pp. 295-302). }\end{array}$ & 1998 \\
\hline
\end{tabular}

Soukoreff, R. W., \& MacKenzie, I. S. (2004).

Towards a standard for pointing device evaluation, perspectives on 27 years of Fitts' law research in

HCI. International journal of human-computer studies, 61(6), 751-789.

Zhai, S. (2004). Characterizing computer input with Fitts' law parameters - the information and noninformation aspects of pointing. International Journal of Human-Computer Studies, 61(6), 791-809.

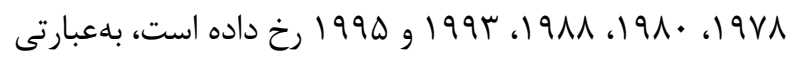
مقالاتى كه در اين سالها شناسايى شدند، بيشترين ارجاع را

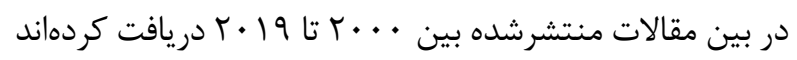

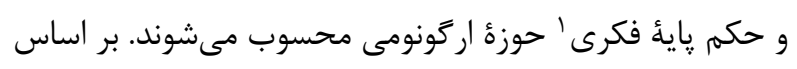

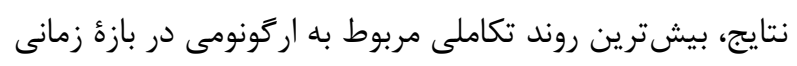

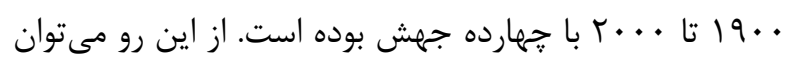

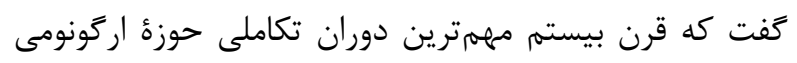
بوده است. همجنين نتايج نشان داد كه بيشتر خاستخاههاى

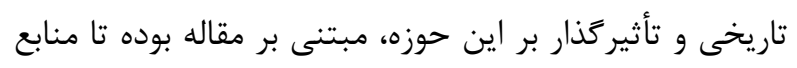

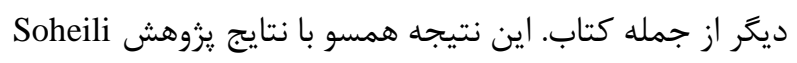

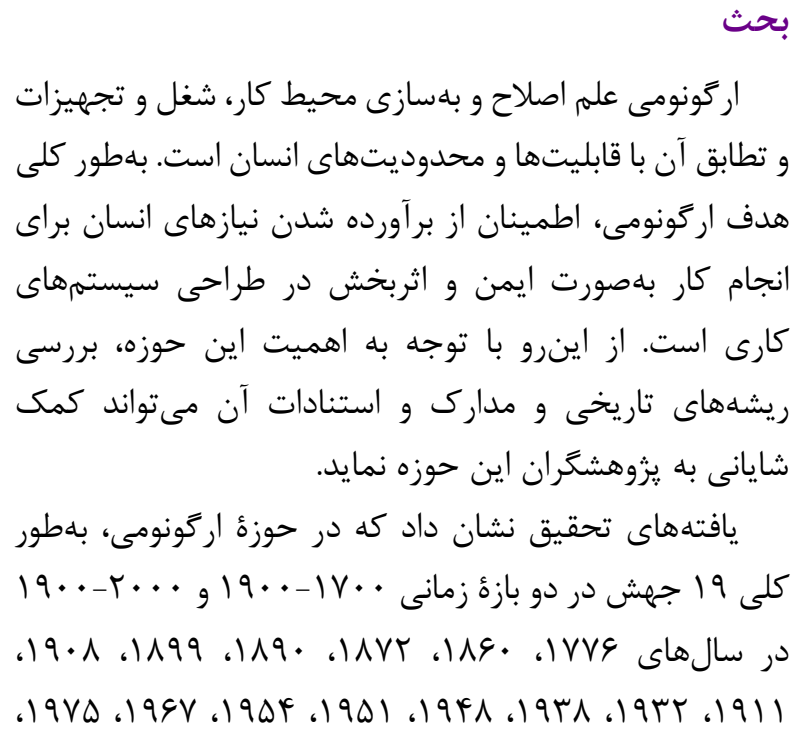

1. Intellectual Base 
تصميمَيرى انسان يويا در حوزههاى مختلف ارائه شده و رابطهٔ

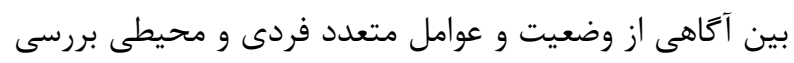

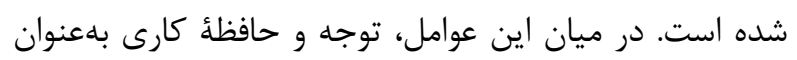

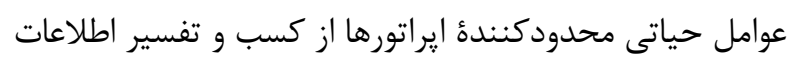

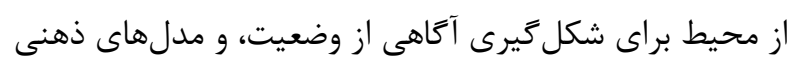

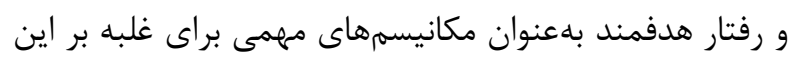

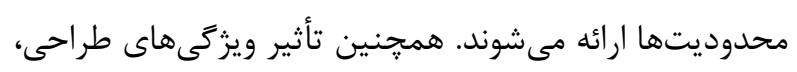

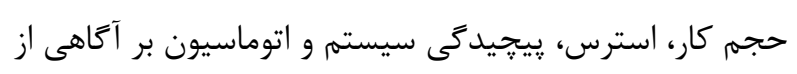

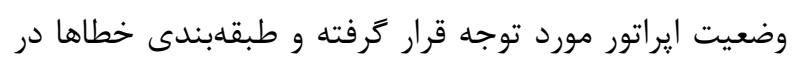

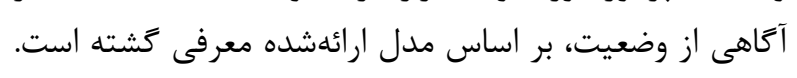

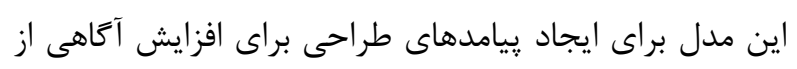

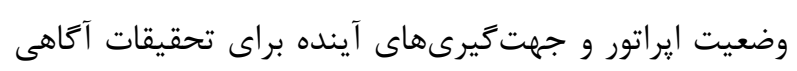

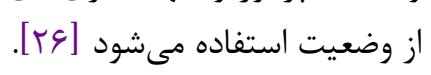

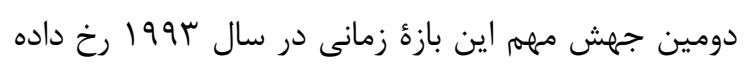

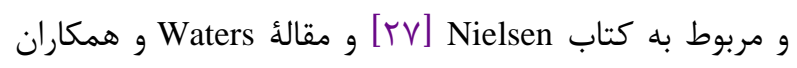

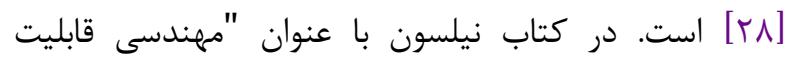

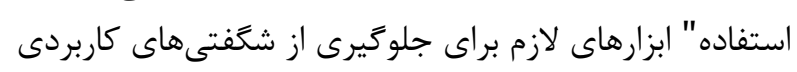

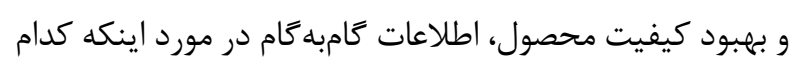

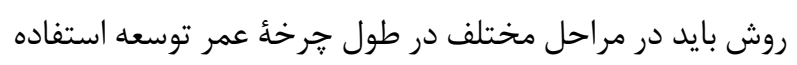

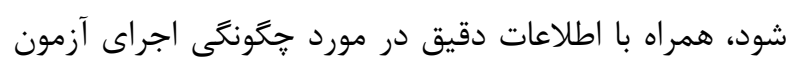

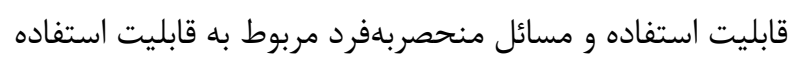

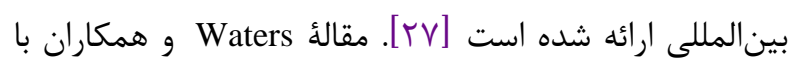

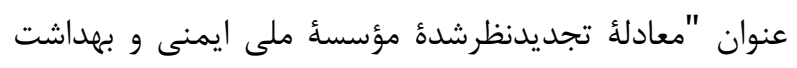

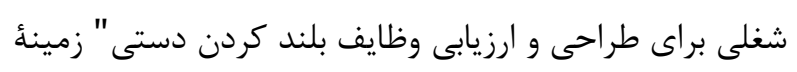

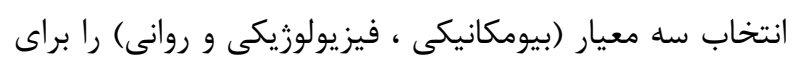

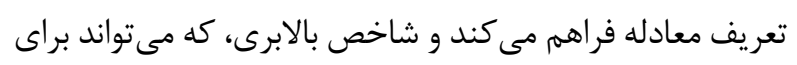

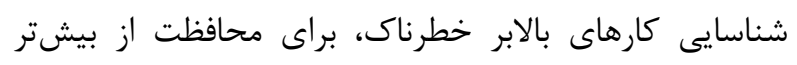

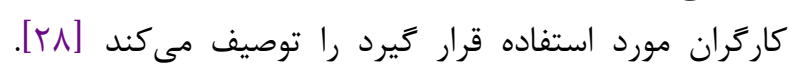

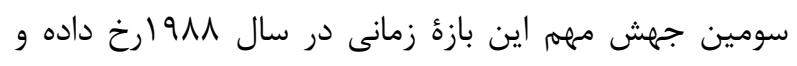

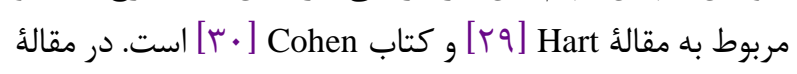
Hart با عنوان "توسعه شاخص باركارى ناسا: نتايج تحقيقات

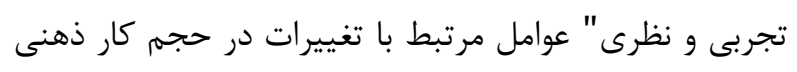

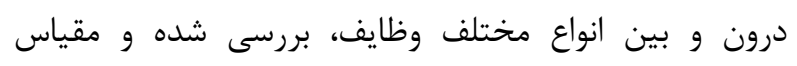

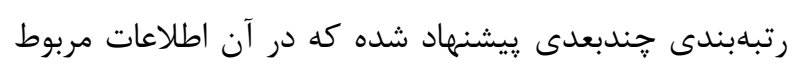

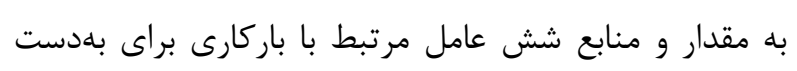

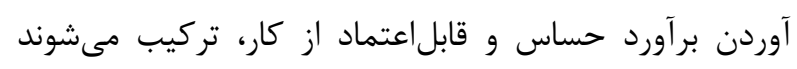

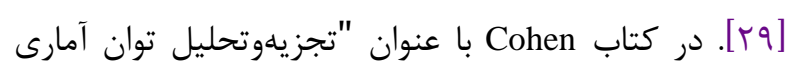

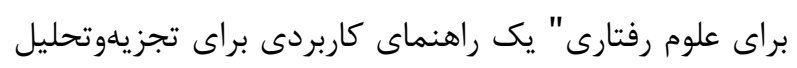

Khasseh gokhtarpour, $[1 \xi](Y \cdot 1 \Delta)$ Khasseh [است

بر اساس نتايج يزوهش بلهطور كلى 19 جهش در رو روند

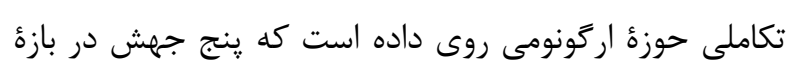

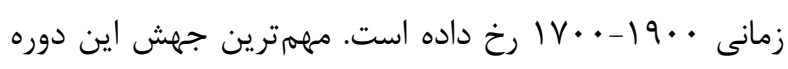

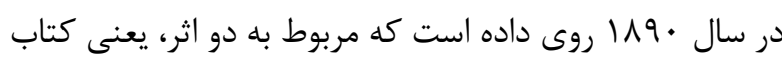

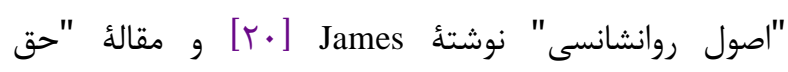

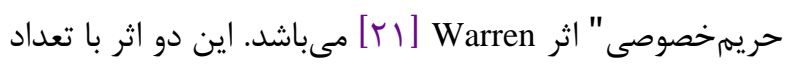

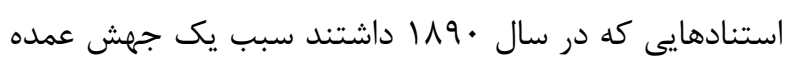

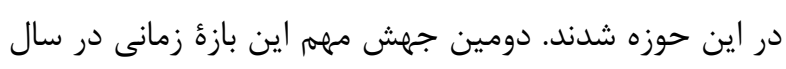

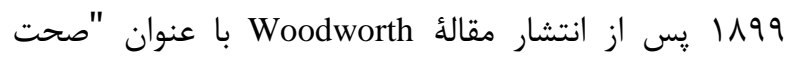

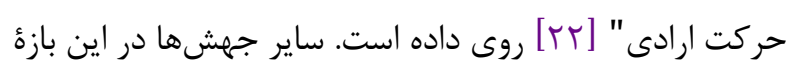

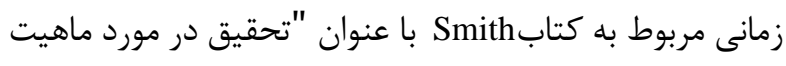

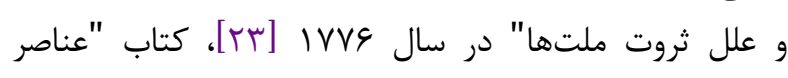

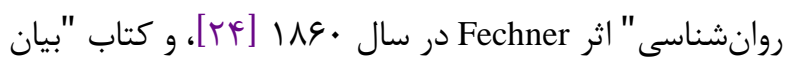

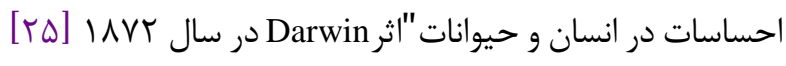

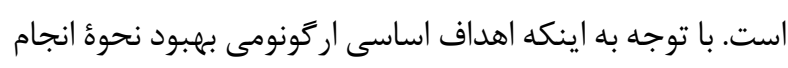

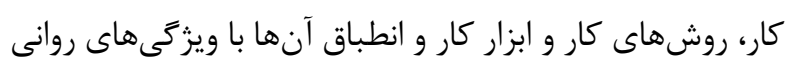

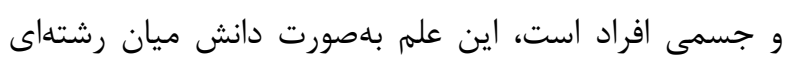

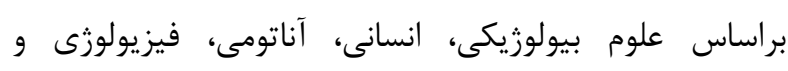

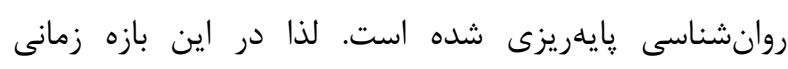

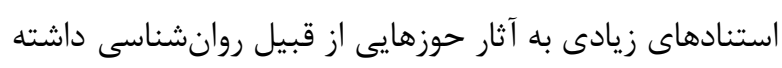

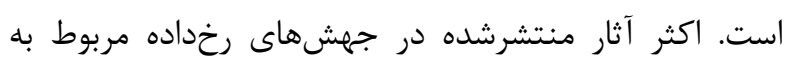

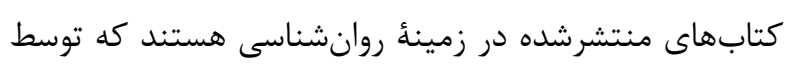

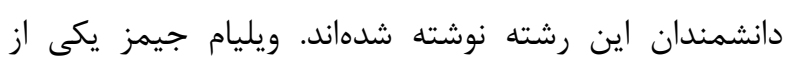

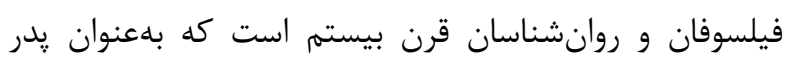

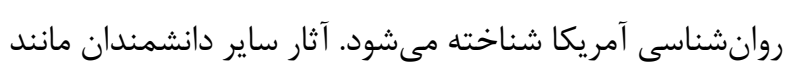

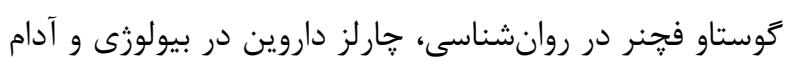

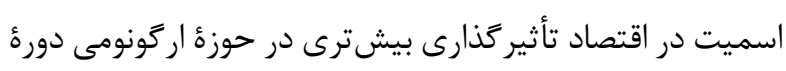

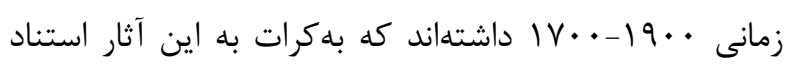
شده است.

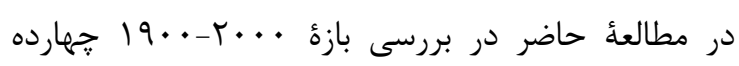

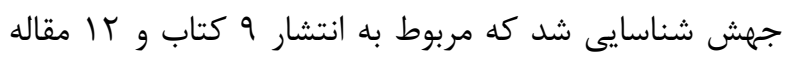

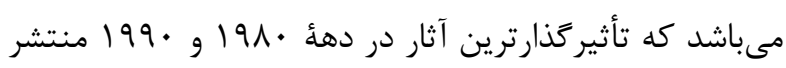

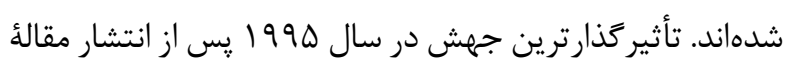
Endsley

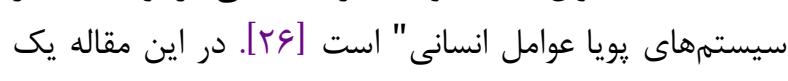

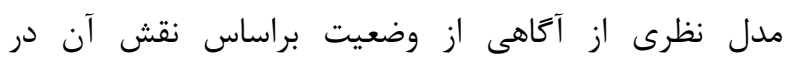


اين ميان دو مقاله با عنوان "به سمت استانداردى براى ارزيابى

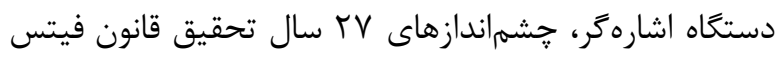

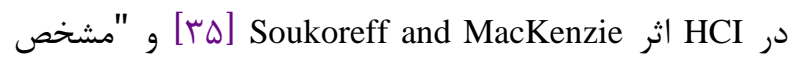

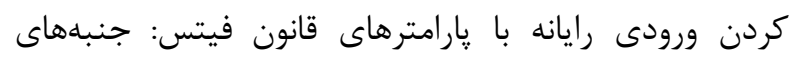

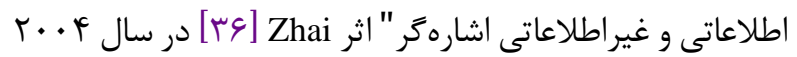

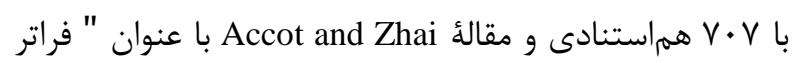

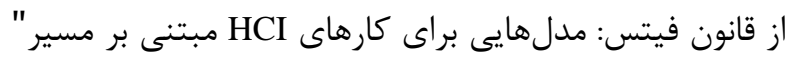

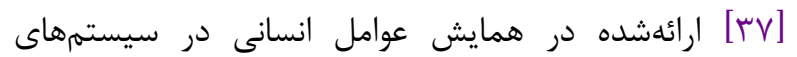

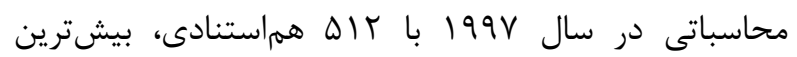

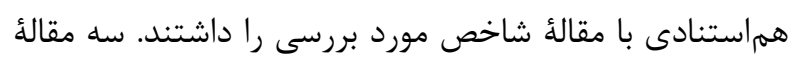

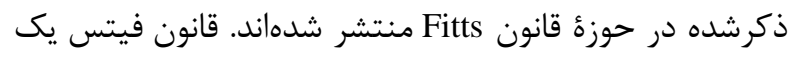
مدل ييشبينى كنندهُ حركت انسان است كه اساساً در تعامل

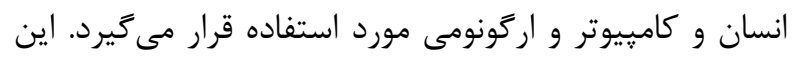

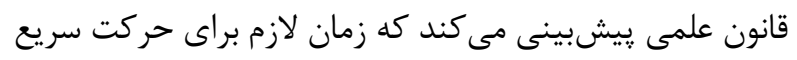

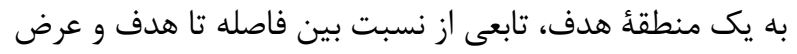

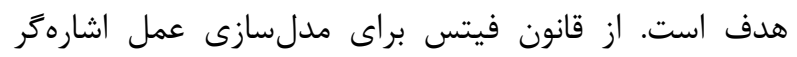

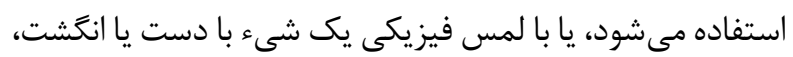

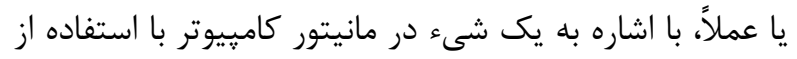

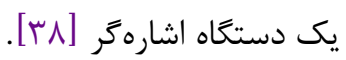

\section{نتيجه كيرى}

بلهور كلى نتايج مطالعأ حاضر در بررسى ريشه تاريخى

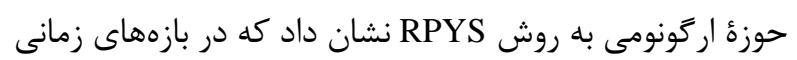

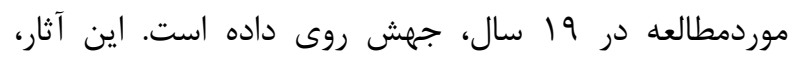

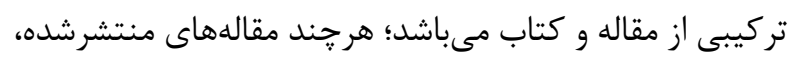

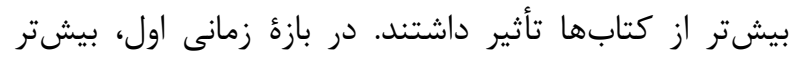

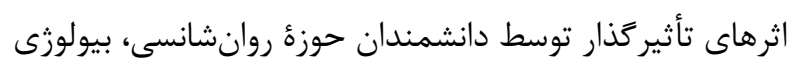

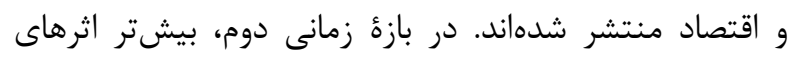

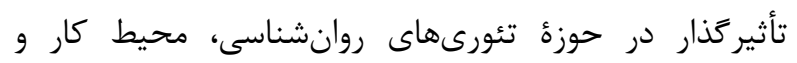

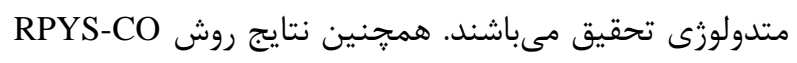

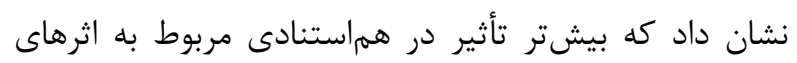

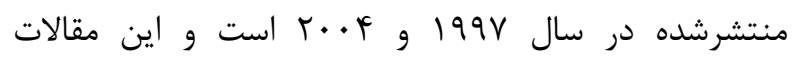

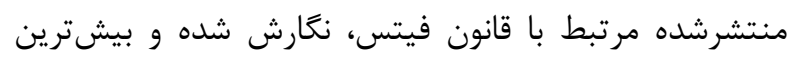

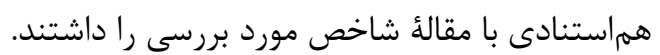

\section{محدوديت هاى و يِيشنهادات براى يزوهش هاى آينده}

اين مطالعه، بهدليل تعداد زياد مدارك موجود در پاييًاههاى

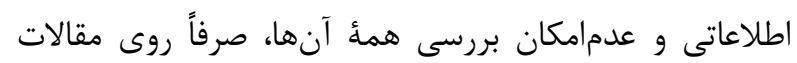

توان آمارى در طراحى يزوهشها است كه ابزارهاى موردنياز

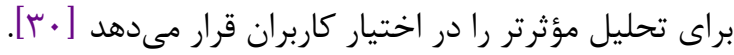

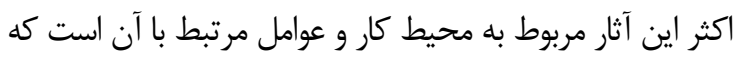

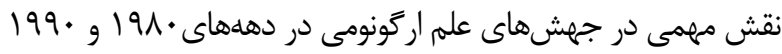

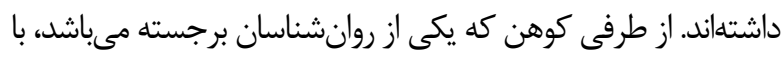

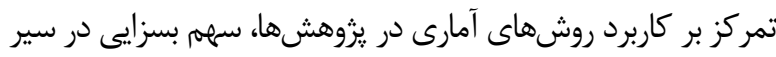

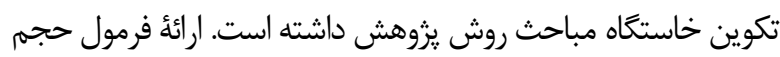

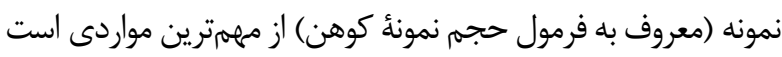

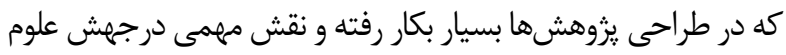

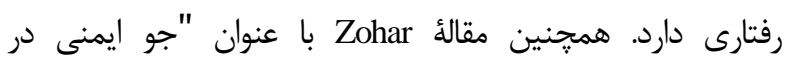

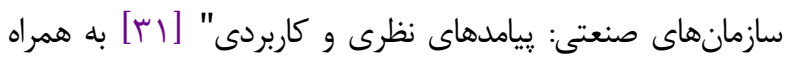

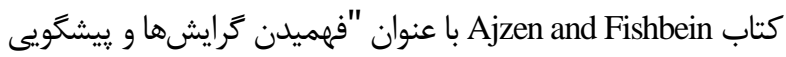
رفتار اجتماعى" در سال •191 بهكرات مورد استناد قرار گرفته و

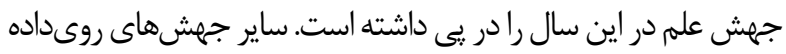

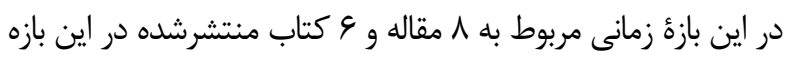
است كه بيشتر اين آثار در حوزهماى تئورىهاى روانشانئاسى، محيط كار و متدولوزى تحقيق مى باشند. نتايج مطالعة حاضر در خصوص بررسى RPYS-CO نشان

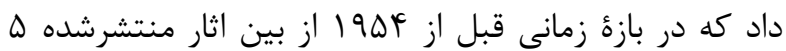
مقاله و يك كتاب، بيشترين همراستنادى با مقالئ شاخص مورد

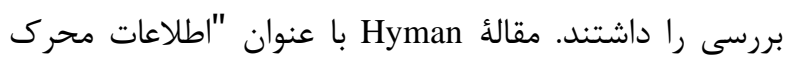

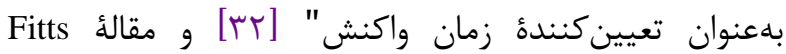

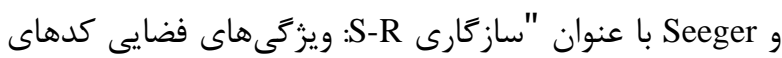

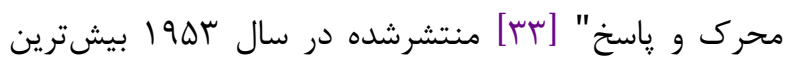

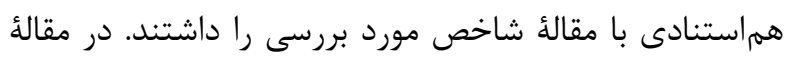
Hyman زمان واكنش به يك محرك بينايى بهعنوان تابعى از باز

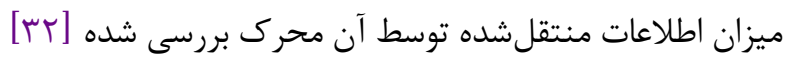

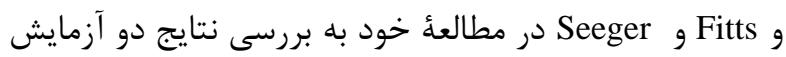
براى نشان دادن سودمندى مفهوم سازگارى ياسخ محرى در در

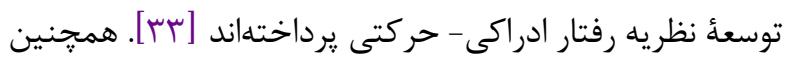

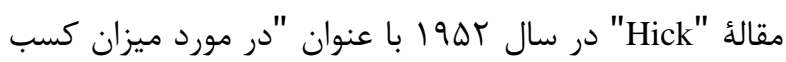

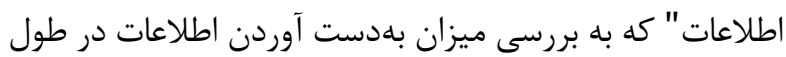

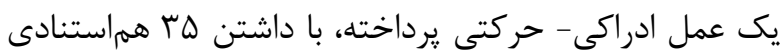

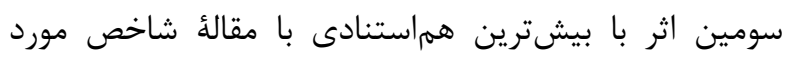
بررسى را داشت [بr].

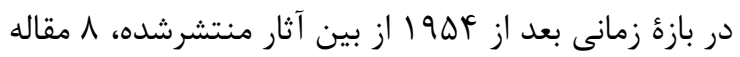

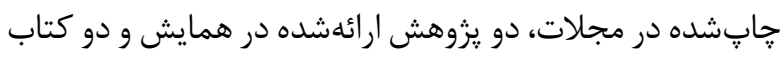

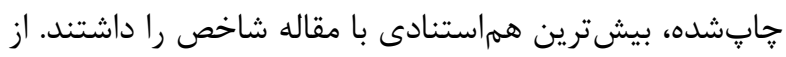




$$
\begin{aligned}
& \text { تقدير و تشكر } \\
& \text { بدينوسيله نويسندًان مقاله، از معاونت تحقيقات و فناورى }
\end{aligned}
$$

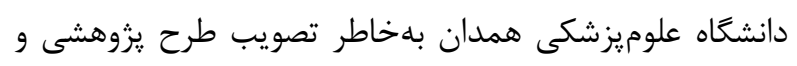

$$
\begin{aligned}
& \text { همكارى در اجراى آن كمال قدردانى و تشكر ران را دارند. } \\
& \text { تعارض منافع } \\
& \text { بين نويسندًان هيجَّونه تعارضى در منافع وجود ندارد. }
\end{aligned}
$$

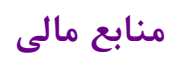

\section{References}

1. Garrido MV, Bittner C, Harth V, Preisser AM. Health status and health-related quality of life of municipal waste collection workers-a cross-sectional survey. J Occup Med toxicol. 2015;10(1):1-7. [DOI:10.1186/s12995-015-0065-6] [PMID] [PMCID]

2. Saha TK, Dasgupta A, Butt A, Chattopadhyay O. Health status of workers engaged in the small-scale garment industry: how healthy are they? Indian J community Med: official publication of Indian Association of Preventive \& Social Medicine. 2010;35(1):179. [DOI:10.4103/0970-0218.62584] [PMID] [PMCID]

3. Hasani S, Mobarak iH, Moghadami Fard Z. The importance of ergonomics in enhancing productivity and improving the performance of Ministry of Health and Medical Education staff. Occup Med Q J. 2013;4(4):92-101.

4. Dul J, Neumann WP. Ergonomics contributions to company strategies. Appl Ergon. 2009;40(4):745-52. [DOI:10.1016/j.apergo.2008.07.001] [PMID]

5. Dempsey P, Wogalter M, Hancock P. Defining ergonomics/human factors. International encyclopedia of ergonomics and human factors, 2nd edn London: Taylor and Francis. 2006:32-5.

6. Mirsepasi N. Strategic management of human resources and labor relations. Tehran: Publication Munir. 2004.

7. Meister D. The history of human factors and ergonomics: CRC Press; 2018. [DOI:10.1201/9781315276069]

8. Mosadeghrad AM. Relationship between nurses' knowledge about ergonomy and their job injuries. J Shahrekord Univ Med Sci. 2004;6.

9. Duriau VJ, Reger RK, Pfarrer MD. A content analysis of the content analysis literature in organization studies: Research themes, data sources, and methodological refinements. Organ Res Methods. 2007;10(1):5-34. [DOI: 10.1177/1094428106289252]
نمايهشده در يايگًا Web of science انجام شده است. از اين رو

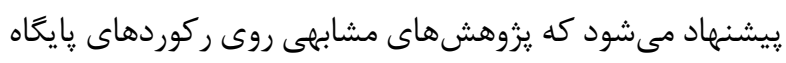

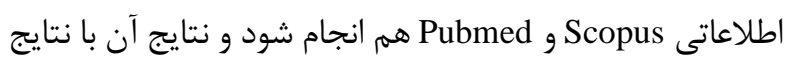
يزوهش حاضر مقايسه گردد. همجنين انجام يك يزوهش

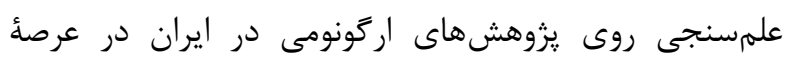

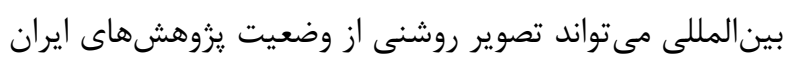
در اين حوزه را ارائه نمايد.

10. Habibi R, Mokhtarpour R, Khasseh AA. Analysis of Evolutionary Trends in Global Entrepreneurship Research using Scientometric Techniques. J Entrep Dev. 2018;10(4):575-94.

11. Khasseh AA, Asghariyan N, Tajedini O, Moosavi A, Ghazizadeh H, Ebrahimzadeh S, et al. 10. Identification and Analysis of the His-torical Origins of Occupational Therapy by Referenced Publication Years Spectroscopy. J Arch. 2019;7.

12. Bornmann L, Marx W. The proposal of a broadening of perspective in evaluative bibliometrics by complementing the times cited with a cited reference $\begin{array}{lll}\text { analysis. J Inf. 2013;7(1):84-8. } & \text {. }\end{array}$ [DOI:10.1016/i.joi.2012.09.003]

13. Mokhtarpour R, Khasseh A. Tracing the Historical Origins of Research Methodology Issues through Referenced Publication Years Spectroscopy (RPYS). J Libr Inf Sci Stud. 2017;24(20):43-58.

14. Marx W, Bornmann L, Barth A, Leydesdorff L. Detecting the historical roots of research fields by reference publication year spectroscopy (RPYS). J Assoc Inf SciTechnol. 2014;65(4):751-64. [DOI: 10.1002/asi.23089]

15. Leydesdorff L, Bornmann L, Marx W, Milojević S. Referenced Publication Years Spectroscopy applied to iMetrics: Scientometrics, Journal of Informetrics, and a relevant subset of JASIST. J Inf. 2014;8(1):162-74. [DOI:10.1016/i.joi.2013.11.006]

16. Soheili F, Khasseh A. Historical Origins of Information Behavior Research by Reference Publication Year Spectroscopy. Iran J Inf Process Manag. 2015;31(1):326.

17. Yao Q, Li X, Luo F, Yang L, Liu C, Sun J. The historical roots and seminal research on health equity: a referenced publication year spectroscopy (RPYS) analysis. Int J Equity Health. 2019;18(1):1-15. [DOI:10.1186/s12939-019-1058-3] [PMID] [PMCID] 
18. Khasseh AA, Mokhtarpour R. Tracing the historical origins of knowledge management issues through referenced publication years spectroscopy (RPYS). J Knowledge Manag. 2016. [DOI:10.1108/JKM-012016-0019]

19. Wray KB, Bornmann L. Philosophy of science viewed through the lense of "Referenced Publication Years Spectroscopy"(RPYS). Scientometrics. 2015;102(3):1987-96. [DOI:10.1007/s11192-014$\underline{1465-6]}$

20. James W. The Principles of Psychology, in two volumes. New York: Henry Holt and Company; 1890.

21. Warren SD, Brandeis LD. Right to privacy., 4, 193. Harv L Rev. 1890;4:193. [DOI:10.2307/1321160]

22. Woodworth RS. Accuracy of voluntary movement. The Psychological Review: Monogr Suppl. 1899;3(3). [DOI: 10.1037/h0092992]

23. Smith A. An inquiry into the nature and causes of the wealth of nations. London: printed for W. Strahan; and T. Cadell; 1776.

24. Fechner G. Elemente der psychophysik Breitkopf u. Härtel.; 1860.

25. Darwin C. The expression of the emotions in man and animals. London: Murray; 1872. [DOI:10.1037/10001000] [PMID] [PMCID]

26. Endsley MR. Toward a theory of situation awareness in dynamic systems. Hum factors. 1995;37(1):32-64. https://doi.org/10.1518/001872095779049543 [DOI: 10.1518/001872095779049499]

27. Nielsen J. Usability engineering: Morgan Kaufmann; 1994. [DOI:10.1016/B978-0-08-052029-2.50009-7]

28. Waters TR, Putz-Anderson V, Garg A, Fine LJ. Revised NIOSH equation for the design and evaluation of manual lifting tasks. Ergonomics. 1993;36(7):749-76. [DOI:10.1080/00140139308967940] [PMID]

29. Hart SG, Staveland LE. Development of NASA-TLX (Task Load Index): Results of empirical and theoretical research. Adv Psychol. 52: Elsevier; 1988. p. 139-83. [DOI:10.1016/S0166-4115(08)62386-9]

30. Cohen J. Statistical power analysis for the behavioral sciences. 2nd ed. ed. Hillsdale, NJ: Lawrence Erlbaum; 1988.

31. Zohar D. Safety climate in industrial organizations: theoretical and applied implications. J Appl Psychol. 1980;65(1):96. [DOI:10.1037/0021-9010.65.1.96] [PMID]

32. Hyman R. Stimulus information as a determinant of reaction time. J Exp Psychol. 1953;45(3):188. [DOI:10.1037/h0056940] [PMID]

33. Fitts PM, Seeger CM. SR compatibility: spatial characteristics of stimulus and response codes. J Exp Psychol. 1953;46(3):199. [DOI:10.1037/h0062827] [PMID]

34. Hick WE. On the rate of gain of information. Q J Exp Psychol. 1952;4(1):11-26 [DOI: $10.1080 / 17470215208416600]$

35. Soukoreff RW, MacKenzie IS. Towards a standard for pointing device evaluation, perspectives on 27 years of Fitts' law research in HCI. Int J Hum-Comput Stud. 2004;61(6):751-89. [DOI:10.1016/j.ijhes.2004.09.001]

36. Zhai S. Characterizing computer input with Fitts' law parameters-the information and non-information aspects of pointing. Int $\mathrm{J}$ Hum-Comput Stud. 2004;61(6):791-809.

[DOI:10.1016/j.ijhes.2004.09.006]

37. Accot J, Zhai S. Beyond Fitts' law: models for trajectory-based HCI tasks. Proceedings of the ACM SIGCHI Conference on Human factors in computing systems; 1997. [DOI:10.1145/258549.258760]

38. Fitts PM. The information capacity of the human motor system in controlling the amplitude of movement. J Exp Psychol. 1954;47(6):381. [DOI:10.1037/h0055392] [PMID]. 